\title{
Influence of Boundary Layer Transition on the Trajectory Optimisation of a Reusable Launch Vehicle
}

\author{
R. Wuilbercq; F. Pescetelli†, E. Miniscił R. E Brown ${ }^{\S}$ \\ Centre for Future Air-Space Transportation Technology, \\ University of Strathclyde, 75 Montrose Street, Glasgow G11XJ, United Kingdom
}

\begin{abstract}
Based on flight experience from the Space Shuttle programme, it is well known that misprediction of the effects of boundary layer transition represents one of the highest technical risks when designing a Reusable Launch Vehicle. Indeed, mis-prediction of the boundary layer behaviour at hypersonic speeds could impinge on the overall survivability of a given design, whereas excessive conservatism in the analyses could result in an overweight vehicle not capable of attaining orbit with a useful payload mass on-board. From the standpoint of conceptual design, it is therefore of paramount importance to develop engineering means of predicting the effects of uncertainty in the behaviour of the boundary layer on the vehicle as far as transition is concerned. Indeed, a robust preliminary analysis should ensure thermal survival of the spaceplane structure and give a measure of confidence in the ability of the conceptual vehicle to maintain sufficiently good controllability during reentry in the presence of possibly asymmetric boundary layer transition. A reduced-order model has been used to evaluate the sensitivity of a particular design of hypersonic reusable launch vehicle to the uncertainty in predicting its aero-thermodynamic behavior that results from variability in the onset of boundary layer transition on its surface, especially when optimising the reentry trajectory of the vehicle. The results of the simulations presented here seem to suggest that the effects of boundary layer transition on the vehicle's performance during re-entry might largely be ameliorated through careful aerodynamic design and appropriate scheduling of the control surface deflections along the vehicle's trajectory.
\end{abstract}

\section{Introduction}

$\mathrm{T}$ HE aerodynamic and aero-thermodynamic loads acting on hypersonic vehicles define the overall performance requirements for various subsystems such as the Thermal Protection System (TPS), the controls and, where appropriate, the propulsion system. Since the aerodynamic heating affects the selection of TPS type, sizing and weight, the reentry trajectory of a Reusable Launch Vehicle (RLV) is generally optimised to achieve an acceptable level of aero-thermal heating. It is well known however that the aerodynamic heating experienced by an RLV during reentry can increase by a factor of two to eight when the boundary layer on the vehicle's surface undergoes a transition from laminar to turbulent flow. ${ }^{1}$

The prediction of boundary layer transition at hypersonic speeds becomes a complicated task for the aerodynamicist since the phenomenon is affected by a very large number of parameters which are difficult to determine or evaluate. Indeed, under reentry conditions, although the flow over the windward surface of an RLV should ostensibly be laminar, the particular form of TPS used, and indeed its manufacturing process, can affect the roughness and temperature at the surface of the vehicle. Premature transition of the boundary layer from laminar to turbulent flow can be triggered by the resultant local increases in the effective Reynolds number of the flow near the surface. In practice, however, the degree to which the surface is roughened, or how the roughness of the surface influences the properties of the flow is, a priori, not always easy to determine (e.g. because of randomly scattered defects on the heat shield as a result of

* PhD Student, Student Member AIAA.

$\dagger$ PhD Student.

${ }^{\ddagger}$ Lecturer.

$\S$ Director and Professor, Senior Member AIAA. 
micro-meteoroid impact, manufacturing defects and tolerances, etc...). The Shuttle Transportation System (STS) was particularly susceptible to this syndrome. For instance, on Shuttle flight STS-50, a measurable amount of extra propellant had to be used to correct the yawing moment that resulted from an asymmetrical boundary layer transition on the leeward surface of the vehicle. During mission STS-114, an unprecedented spacewalking repair had to be carried out to remove the threat posed by two forward-located gap fillers which could potentially have affected the integrity of the TPS in the case of an early onset of transition during re-entry. ${ }^{2}$

Because of these issues, the optimisation of the reentry path followed by an RLV from its high-angle-ofattack re-entry down to its landing site is highly dependent on accurate prediction of the transition from laminar to turbulent flow. The validity and robustness of the results of the optimisation are thus intrinsically linked to the accurate prediction of laminar-turbulent transition. In the National Aerospace Plane Review published by the Defense Science Board in 1988, it was stated that estimates of the transition location could range from $20 \%$ to $80 \%$ along the body length and could thus affect the designed vehicle Gross Take-Off Weight (GTOW) by a factor of two or more. Indeed, since fully turbulent analysis does not permit accurate predictions of thermal gradient and results in excessive TPS weight penalties, and since fully laminar analysis can lead to under-design of the TPS with resultant impact on vehicle survivability in the hypersonic flight environment, it is of paramount importance that numerical optimisation tools represent the phenomenon of boundary layer transition properly in order to provide confidence in the robustness of the design of future RLVs from the earliest stages of their conceptual evolution.

The objective of the present work is however not to identify the primary drivers of uncertainty for the boundary layer transition phenomenon, nor to make any major improvements in the accuracy with which laminar-turbulent transition can be predicted. Instead, the aim is to explore and illustrate the properties that would be required of a robust engineering methodology in which any sensitivity to the uncertainties in the flow physics that are associated with boundary layer transition can be accomodated, and to demonstrate a prototype of such a process as applied to the preliminary design of a typical Reusable Launch Vehicle.

\section{Background}

$\mathrm{T}^{\mathrm{T}}$ is well recognised that the modelling of boundary layer transition is one of the most difficult challenges Iin fluid dynamics. No theory yet exists that can be used to accurately predict transition at hypersonic speeds. ${ }^{3}$ Indeed, most of the transition prediction techniques available for design are in the form of empirical correlations, and these, whether obtained in ground-based test facilities or in flight, have a limited range of applicability. Engineering methods often assume that transition occurs at a Transition Point (TP). The flow is assumed laminar upstream of this point, and fully turbulent downstream. However, for the accurate prediction of the skin friction and heat transferred to a body, knowledge of the transition Reynolds number $R e_{T}$ is critical. Since limited theoretical guidance exists, the appropriate value of $R e_{T}$ for a given situation is generally obtained from experimental data. Any application outside the existing database, for instance for preliminary design purposes, is fraught with difficulty and requires a number of assumptions, the validity of which is always highly dependent on the expertise of the designers.

The Reynolds number at transition is usually dependent on a very large number of parameters which are either assumed, can be predicted, or are "known unknowns". For instance, the Mach number at the edge of the boundary layer, $M_{e d g e}$, has a strong influence on the stability of the laminar boundary layer and, by extension, on the transitional Reynolds number $R e_{T}$. Three-dimensionality in the flow can also have a strong effect on laminar-turbulent transition. ${ }^{4}$ Indeed, it was shown that flows over two-dimensional flat plates have higher transition Reynolds numbers than cones at the same free-stream conditions. ${ }^{5}$ Hence, the Reynolds number at transition can be expressed in a functional form depending on a range of parameters, for example

$$
\operatorname{Re}_{T}=f\left(M_{\text {edge }}, \alpha, T_{\text {wall }}, k_{R}\right)
$$

where $T_{\text {wall }}$ is the wall temperature and $k_{R}$ is a parameter expressing the roughness of the surface.

The physical models used to characterize these parameters are also subject to errors and uncertainties. These uncertainties are usually a consequence of miscellaneous combined factors that include inherent assumptions, lack of knowledge, lack of corroborative experimental and flight data, application of models beyond their validated range, and other sources of errors that have been accepted, perhaps if only in the 
interest of developing a convenient and rapid design methodology. The physical models that are used are thus often subject to a large inherent uncertainty - which can lead in practice to large discrepancies between predicted and in-flight values of parameters such as Peak Heat Flux (PHF), Integrated Heat Load (IHL) or viscous drag.

\section{Aero-thermodynamic Model}

B EARING in mind these provisos, the advantage of reduced-order models for preliminary design is of B course the ease with which parametric variations in the properties of the system can be explored. Our reduced-order model, dubbed HyFlow, is an engineering level aero-thermodynamic tool for predicting the aerodynamic and aero-thermal environments of general three-dimensional vehicle configurations. The associated reduction in terms of physical realism of this approach, especially compared to modern numerical techniques such as Computational Fluid Dynamics (CFD) and Direct Simulation Monte Carlo (DSMC), is offset entirely, in many practical applications, by the very low computational cost and parametric flexibility of the approach. The Hyflow model uses an unstructured triangular mesh to define the geometry of the vehicle, and proceeds to calculate the aerodynamics, thermal loads, propulsive system effects and so on using the formalism described below. In assessing the simplifications and assumptions inherent in the methodology, it should be borne clearly in mind that the purpose of the present analysis is to examine the effect of uncertainties in the behaviour of the boundary layer on the robustness with which the re-entry trajectory of a Single-Stage to Orbit Reusable Launch Vehicle such as that shown in Fig. 1 can be designed.

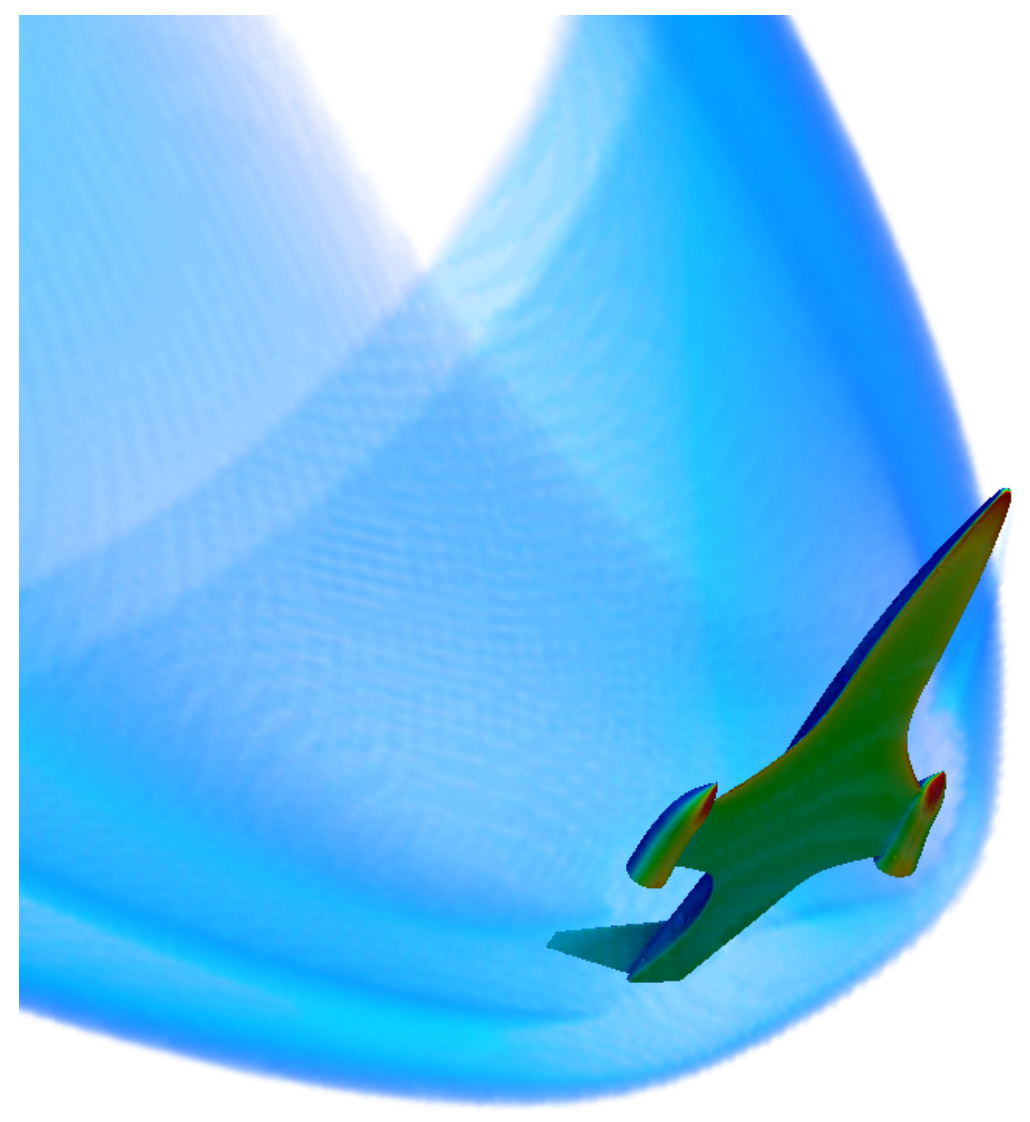

Figure 1: The CFASTT-1 Reusable Launch Vehicle shown during a simulated re-entry, travelling at Mach 24 and an altitude of $115 \mathrm{~km}$. The shock-wave structure and thermal loads illustrated in this figure were calculated using the DSMC technique. ${ }^{6}$ 


\section{III.A. Aerodynamic Model}

\section{III.A.1. Inviscid Aerodynamics}

In the present work, modified Newtonian theory is used to calculate the inviscid aerodynamic environment of our representative reentry vehicle, starting from a high angle-of-attack reentry and ending at the onset of the Terminal Area Energy Management (TAEM) regime at supersonic speed. The use of this local inclination method can be justified by the nature of the reentry trajectory of our RLV. Indeed, as a means to reduce the total heat load along the trajectory, the vehicle maintains a high angle of attack during a significant portion of the descent, and its aerodynamics thus resemble very closely those of a blunt body for most of the duration of its flight. Assuming a Newtonian flow, the pressure exerted on the vehicle is then solely due to the total loss of momentum of the fluid in the direction normal to the vehicle's surface. The local pressure coefficient $C_{p}$ at any point on the body is then given by: ${ }^{4}$

$$
C_{p}=\left\{\begin{array}{l}
C_{p, \max } \cos ^{2} \theta, \text { if } \theta<\frac{\pi}{2} \\
0, \text { if not }
\end{array}\right.
$$

where $\theta$ represents the angle between the free stream velocity vector $\vec{V}_{\infty}$ and $\hat{n}$ is the body inward unit normal vector. The modified Newtonian theory used in the Hyflow model accounts for the pressure loss across the normal shock that is invariably located in the flow upstream of the vehicle, and uses the Rayleigh-Pitot tube formula to relate the stagnation pressure behind a normal shock wave $p_{0,2}$ to the free-stream pressure $p_{\infty}$ as

$$
\frac{p_{0,2}}{p_{\infty}}=\left(\frac{1-\gamma+2 \gamma M_{\infty}^{2}}{\gamma+1}\right) \times\left(\frac{(\gamma+1)^{2} M_{\infty}^{2}}{4 \gamma M_{\infty}^{2}-2(\gamma-1)}\right)^{\gamma /(\gamma-1)}
$$

The maximum pressure coefficient in Eq. 2 is thus given by

$$
C_{p, \max }=\frac{2}{\gamma M_{\infty}^{2}} \times\left(\frac{p_{0,2}}{p_{\infty}}-1\right)
$$

\section{III.A.2. Viscous Effects}

As the Mach number is increased, the strong shock that is always found upstream of the vehicle moves closer to the body while the boundary layer grows rapidly. At hypersonic Mach numbers, viscous interactions between the outer inviscid flow, the shock, and the boundary layer become significant and therefore cannot be neglected. Our reduced-order model accounts for viscous effects by finding the trajectories of the streamlines across the surface of the vehicle, then integrating along the streamlines to find the local Reynolds number $R e^{*}$ and hence the local skin friction coefficient. In the present work, the relationships used to calculate the skin friction coefficients, denoted $C_{f, l a m}$ and $C_{f, t u r b}$ for laminar or turbulent flow respectively, are derived from those for compressible flow over a flat plate:

$$
\begin{aligned}
C_{f, \text { lam }} & =K_{g} \frac{0.664}{\left(R e^{*}\right)^{0.5}} \times f_{1} \\
C_{f, \text { turb }} & =K_{g} \frac{0.02296}{\left(R e^{*}\right)^{0.139}} \times f_{2}
\end{aligned}
$$

where $f_{1}$ and $f_{2}$ are factors added to account for compressibility effects, $K_{g}$ is a Mangler factor used to correct the results from two-dimensional flat plate theory to account for three-dimensionality in the flow $\left(K_{g}\right.$ is set equal to $\sqrt{3}$ for laminar flows and 1.15 for turbulent flows), and $R e^{*}$ is the local Reynolds number evaluated at a reference temperature $T^{*}$ which is representative of the temperature inside the boundary layer. The Smart-Meador reference temperature is used in this work for both laminar and turbulent flows. ${ }^{7}$ Temperature effects are included by assuming a wall temperature $T_{\text {wall }}$, herein set to $300 \mathrm{~K}$, and using Sutherland's viscosity law in order to account for the variation of viscosity with temperature, using standard temperature and pressure as the reference values. 


\section{III.A.3. Aerodynamic Forces and Moments}

The total force and moment acting on the vehicle can then be calculated by integrating the pressure and shear force distributions over the surface area of the vehicle. Since our reduced-order model uses a panel-based approximation to the geometry of the vehicle, this integration is approximated numerically by performing a finite summation over the total number of panels $N$ that approximate the shape of the vehicle.

The aerodynamic force acting on panel $i$ is given by

$$
\hat{F}_{i}=\left\{Q_{\infty}\left(C_{p}\right)_{i} \hat{n}_{i}+\left(Q_{\text {ref }} C_{f}\right)_{i} \hat{s}_{i}\right\} \quad A_{i}
$$

where the first term represents the inviscid contribution from Eq. 2 on the preceding page, and the second the viscous contribution from Eq. 5 on the previous page evaluated at the centroid of each panel, area $A_{i}$, comprising the approximation to the shape of the vehicle. $\hat{S}_{i}$ is the unit vector in the direction of the shear force on the $i$-th panel and is approximated as

$$
\hat{s}_{i}=\left(\hat{n}_{i} \times \frac{\vec{V}_{\infty}}{\left\|\vec{V}_{\infty}\right\|}\right) \times \hat{n}_{i},
$$

$Q_{\infty}=\frac{1}{2} \rho_{\infty}\left\|\vec{V}_{\infty}\right\|^{2}$ is the free-stream dynamic pressure and $Q_{r e f}$ is the local reference dynamic pressure at the edge of the boundary layer. The overall aerodynamic force acting on the vehicle is then approximated by

$$
\hat{F}=\sum_{i=1}^{N} \hat{F}_{i}
$$

and, similarly, the aerodynamic moment about the centre of mass $\hat{x}_{c}$ of the vehicle is approximated by

$$
\hat{M}=\sum_{i=1}^{N} \hat{F}_{i} \times\left(\hat{x}_{i}-\hat{x}_{c}\right)
$$

where $\hat{x}_{i}$ is the centroid of panel $i$.

\section{III.B. Aerodynamic Heating Model}

HyFlow evaluates the acreage heating based on the flat plate reference temperature method as described in section III.A on the preceding page for evaluating the skin friction. It then uses the well-known Reynolds analogy, taking advantage of the similarity between friction and heat transfer mechanisms, in order to estimate the Stanton number, $S t$, and therefore the local convective heating rate $\dot{q}_{c o n v}$ from the skin friction estimates.

Since the method based on the Reynolds analogy and the underlying streamline tracing algorithm is inherently invalid at the stagnation features within the flow, a mix of methods must be employed. HyFlow uses a modified version of the Fay-Riddell formula to calculate the convective heating rate for general threedimensional stagnation points. The method is described in detail by DeJarnette et al. ${ }^{8}$ The heat flux at a general three-dimensional stagnation feature, for a wall which is cold relative to the flow (which is usually the case for hypersonic reentry) is related to the heating rate at an axisymmetric point by the following relationship:

$$
\dot{q}_{\text {stag }}=\sqrt{\frac{1+k}{2}} \dot{q}_{a x i}
$$

where the parameter $k$ is the ratio of the principal radii of curvature of the vehicle surface at the location of the stagnation feature, estimated using the algorithm of Cohen-Steiner et al. ${ }^{9}$ (so that $k=R_{1} / R_{2}$ where $R_{2} \geq R_{1}$ ), and $\dot{q}_{a x i}$ is given by the well-known Fay-Riddell formula. ${ }^{10}$

\section{III.C. Transition Model}

In order to define accurately the aero-thermodynamic environment of the RLV during its re-entry into the atmosphere, a criterion must be devised for defining when the transition of the boundary layer from the laminar state to a turbulent state occurs. A simple empirical correlation, based on data for sharp cones at 
zero angle of attack, and used by Bowcutt and Anderson in a study of hypersonic waveriders, ${ }^{11}$ is used in HyFlow to predict the onset of transition on those vehicle surfaces that are smooth:

$$
\log _{10}\left(R e_{T}\right)=6.421 \exp \left(1.209 \times 10^{-4} M_{\text {edge }}^{2.641}\right)
$$

where $R e_{T}$ is the streamline Reynolds number at which transition takes place and $M_{\text {edge }}$ is the Mach number at the edge of the boundary layer. The entropy at the edge of the boundary layer is simply assumed to be the post-normal shock entropy which fixes the flow state at the edge of the boundary layer. The value of $M_{\text {edge }}$ is therefore influenced by the free-stream conditions $T_{\infty}$ and $P_{\infty}$.

\section{Robustness and Uncertainties}

$\mathrm{M}$ $\mathrm{UCH}$ still remains to be understood regarding the various phenomena that could potentially trigger the early onset of transition in hypersonic flight. As discussed in section II on page 2, when and if boundary layer transition occurs depends on many coupled parameters. ${ }^{12}$ The effect of only a small subset of these parameters is considered in this work. Under normal conditions, transition should usually occur symmetrically on both left and right sides of the vehicle at about Mach 8 during a nominal entry trajectory for a Shuttle-sized RLV. Many parameters can potentially cause premature transition, however. Statistical analysis of the reentry flight experience of the Space Shuttle has shown that early transition, i.e. at greater than Mach 10.9, occurred on $20 \%$ of flights, and that transition was asymmetric in $60 \%$ of those cases. Nevertheless, for lower Mach numbers, the probability of asymmetric transition reduced to only $10 \% .{ }^{13}$

\section{IV.A. Transition Uncertainty}

Laminar-turbulent transition intrinsically encompasses two types of uncertainty. The first one is the stochastic uncertainty (also called random uncertainty) that is associated with inherent variations in the physical system or its environment. The windward surface of future RLVs will, in all likelihood, be comprised of overlapping, sharp-cornered panels made out of very-high-temperature resistant materials. Under repeated use, these panels might eventually yield a pattern of asperities with edges that protrude into the local flow. ${ }^{14}$ These protective panels might also bow outwards or inwards under the aerothermal loads of reentry, and this could provoke premature transition to turbulent flow which could, in turn, lead to downstream surfaces being subjected to a more severe heating environment than might otherwise be the case. Another major source of uncertainty for hypersonic vehicles is often the extent to which the condition of the TPS surface has deteriorated in flight prior to transition, or indeed prior to or during launch as a result of manufacturing and maintenance errors. Finally, boundary layer transition comprises a second type of uncertainty which is epistemic, or in other words is the result of the lack of knowledge that arises from the use of inadequate physical models. Since, as discussed in section II on page 2, there is no universal value for the transitional Reynolds number $R e_{T}$, the inherent uncertainty in predictions that results from the evaluation of this parameter, for instance through correlations such as Eq. 11 on the previous page, needs to be be quantified in order to obtain a measure of the robustness of the design process.

\section{IV.B. Uncertainty Modelling}

When optimizing the trajectory of an RLV during atmospheric entry, small differences in the predicted atmosphere through which the vehicle descends, or for instance in the manufacturing of the TPS material, will lead to scatter in the results of predictions of the reentry trajectory. Therefore, in order to assess the robustness, or in other words the feasibility in practice of a proposed reentry trajectory, a number of model parameters need to be considered as uncertain, and the results of the simulations of the trajectory in the presence of this uncertainty must then be interpreted in a statistical sense.

\section{IV.B.1. Roughness-Induced Transition}

Taking inspiration from the work of Ryan et al, ${ }^{15}$ our reduced-order model can simulate some of the effects of roughness-induced boundary layer transition, such as the creation of asymmetric aerodynamic loads and increased aerodynamic heating, by introducing an isolated element of surface roughness onto the Outer Mould Line (OML) of any arbitrary three-dimensional vehicle configuration. The location of the roughness element can either be imposed, or randomized within a prescribed region of interest on the surface of the 


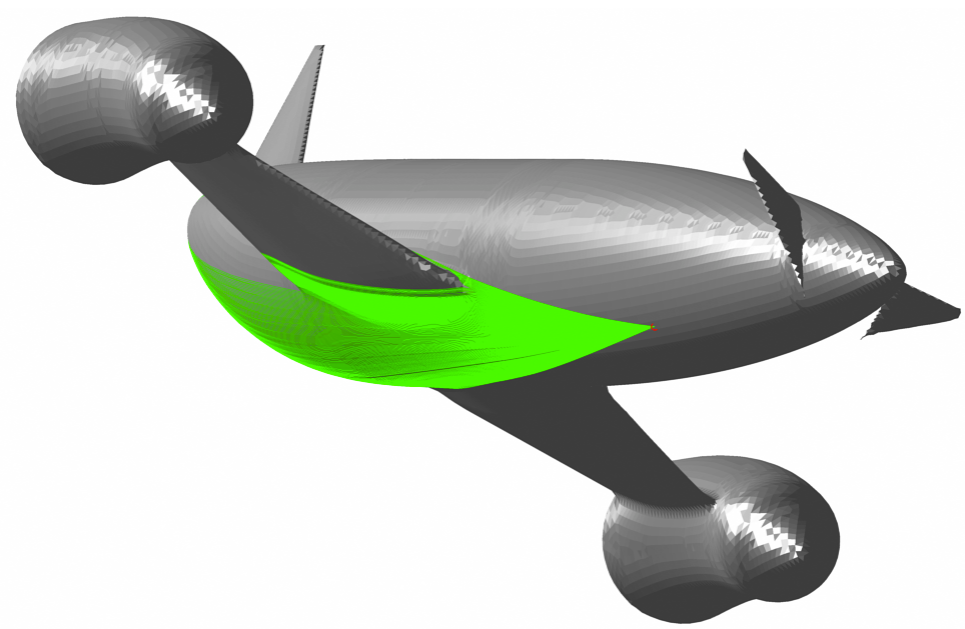

Figure 2: A set of streamlines is used to define the extent of the turbulent wedge region that results from the presence of an element of surface roughness on the lower forward fuselage of the Skylon SSTO vehicle.

vehicle. Likewise, the severity of the element of roughness, in terms of its influence on the boundary layer properties, can either be prescribed or randomised. The extent of the region of spread of the turbulence downstream of the roughness, i.e. the so-called "turbulent wedge", depends on the conditions at the edge of the boundary layer at the location of the roughness element; namely the density $\rho_{k_{r}}$, the velocity $U_{k_{r}}$, and the viscosity $\mu_{k_{r}}$ of the flow. The turbulent wedge that results from an isolated element of surface roughness has its apex at the location of the element and expands downstream along the surface of the vehicle. Within the turbulent wedge region, a fully turbulent boundary layer is assumed, and the apex half-angle $\phi_{\text {wedge }}$ of the wedge is modelled by the empirical relationship ${ }^{16}$

$$
\phi_{\text {wedge }}=3^{-3 / 2} \times \sqrt{2} \times \frac{1}{M_{\infty}}
$$

where $M_{\infty}$ is the free-stream Mach number.

The algorithm used by HyFlow to define a turbulent wedge on the surface of the vehicle starts by tracing its leading edge by marching downstream from its apex and calculating the boundary between the laminar flow upstream and the turbulent flow within the wedge. This is done by offsetting locally the leading edge of the wedge, with respect to the local streamline, by the wedge apex angle calculated according to Eq. 12. An example of the end result of this process is shown in Fig. 2. The region of the surface mesh that lies beneath the turbulent wedge is then identified, and the flow is assumed to be turbulent above all panels that lie within that region. The creation of turbulent wedges is triggered during trajectory integration when the local roughness Reynolds number,

$$
R e_{k_{r}}=\left(\frac{\rho_{k_{r}} \times U_{k_{r}} \times h_{\text {wedge }}}{\mu_{k_{r}}}\right)
$$

satisfies the criterion $R e_{k_{r}} \geq 300$, where $h_{\text {wedge }}$ is the height of the surface roughness element that was responsible for causing the turbulent wedge in the first place. The number of turbulent wedge regions, as well as the geometrical extent of each such region on the vehicle surface, is then updated at each time-step throughout the re-entry simulation. The effect of each wedge on the local aerodynamic loads and heat flux is accounted for until the smooth-surface turbulent region merges with and subsumes the roughnessinduced turbulence (see Fig. 3 on the following page). The effect of asymmetric transition through the mechanism of turbulent wedge formation is to temporarily unbalance the aerodynamic forces on the vehicle. The resultant aerodynamic moments must then be counteracted by appropriate deflection of the control surfaces, as described in section V.C on page 10. The trimming of the aerodynamic moments in this way increases the overall drag on the vehicle with concomitant effects on its re-entry trajectory (see section V on page 9). In addition, the local increases in aerodynamic heating that result from the presence of surface roughness can be accommodated into the constraints that are applied within the optimisation procedure described in section $\mathrm{V}$ on page 9. 


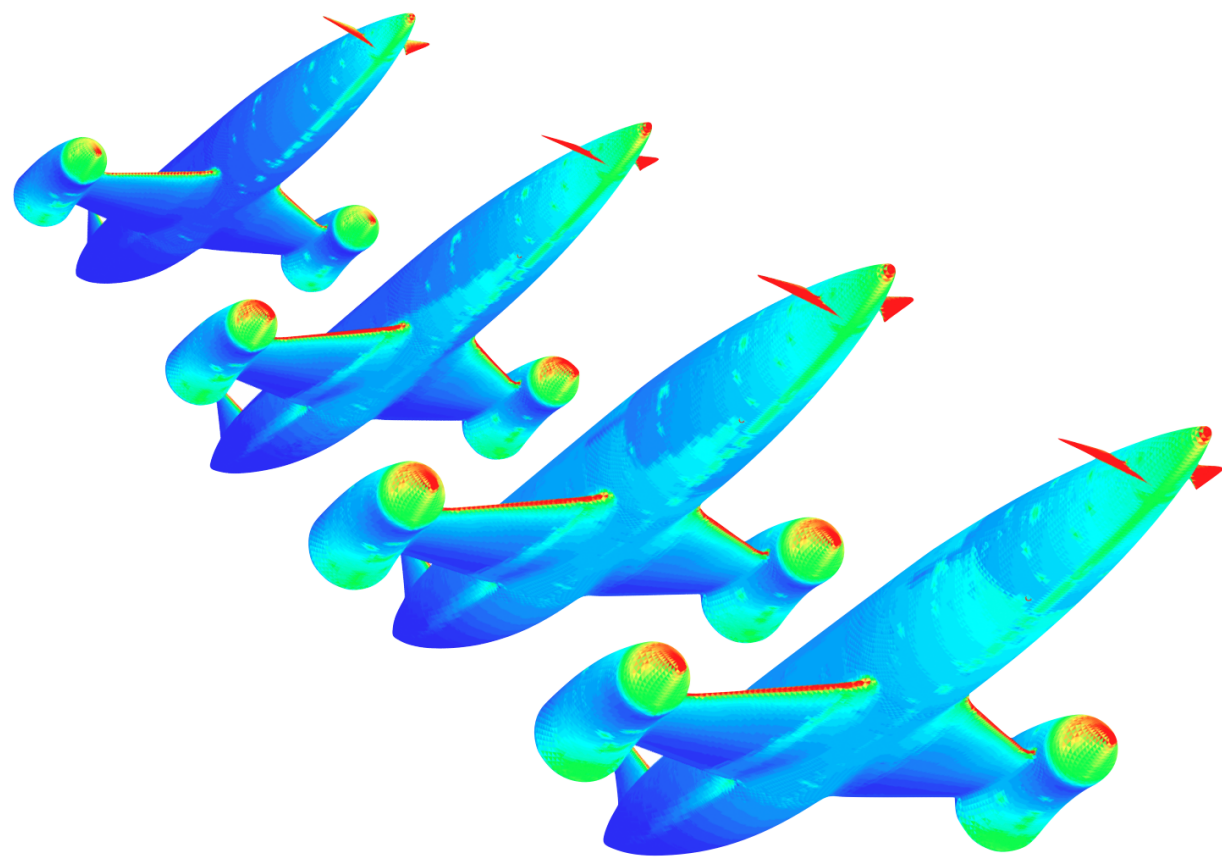

Figure 3: An illustration of the effect of the turbulent wedge model on the HyFlow-predicted surface heating of the Skylon SSTO vehicle at various points along its reentry trajectory. The calculation starts from a fully laminar flow (top left), before a turbulent wedge appears (middle). The wedge develops, subsequently to merge into the "smooth-surface" turbulent flow that envelops most of the surface of the vehicle towards the end of its trajectory (bottom right).

\section{IV.B.2. Atmospheric Model}

Natural variations in the atmospheric pressure $P_{\infty}$ and temperature $T_{\infty}$, through their effect on the flow conditions at the edge of the boundary layer, are major sources of uncertainty when predicting the onset of laminar-turbulent transition (see section III.C on page 5). Uncertainty in these parameters of course has an impact on the inherent uncertainty in many other parameters within the atmospheric model, for instance the speed of sound $a_{\infty}$ and the density $\rho_{\infty}$ through the gas equation of state. By perturbing the outputs of the atmospheric model, uncertainty is generated within the aerodynamic model through its inputs, such as the Mach number $M_{\infty}$ and dynamic pressure $Q_{\infty}$. By this means, the atmospheric uncertainty is propagated into the various models for boundary layer transition that are embedded within HyFlow.

The uncertainty in the properties of the atmosphere is represented in HyFlow by treating the atmospheric temperature profile, $T_{\infty}(h)$, where $h$ denotes altitude above sea level, as a random variable. More specifically, if the nominal temperature profile within the atmosphere is denoted as $T_{\infty}^{n o m}(h)$, then a representation of the temperature profile in the presence of uncertainty is constructed as

$$
T_{\infty}(h)=T_{\infty}^{n o m}(h)+\varepsilon(h) S(h)
$$

In this expression, $\varepsilon$ is an error bound function which captures the statistical variance of the temperature with altitude. This function can, at least in principle, be determined from measurement. For present purposes, and in the absence of better information, $\varepsilon$ is modelled very simply by linear interpolation between assumed bounds $\varepsilon_{L}$ and $\varepsilon_{U}$ on the uncertainty at the lower $(h=0)$ and upper $\left(h=h_{U}\right)$ edges of the atmosphere, respectively, so that

$$
\varepsilon(h)=\left(1-h / h_{U}\right) \varepsilon_{L}+\left(h / h_{U}\right) \varepsilon_{U}
$$

The function $S(h)$ captures the detailed statistical form of the distribution of the temperature perturbations about the nominal variation with altitude. For present purposes it is assumed simply that $S(h)$ is a uniform distribution on the interval $[-1,1]$. As will be shown later, this assumption, although appearing at first sight to be physically rather simplistic, is of great utility when testing the robustness of a design as opposed 
to when performing a direct simulation of its likely performance. Finally, the variability that is introduced into the free-stream temperature using this approach are propagated into the remainder of the atmospheric model by imposing the ideal gas law and barostatic equilibrium. A typical set realisations of the variation of the atmospheric properties with altitude generated using this approach is shown in Fig. 4 on the next page.
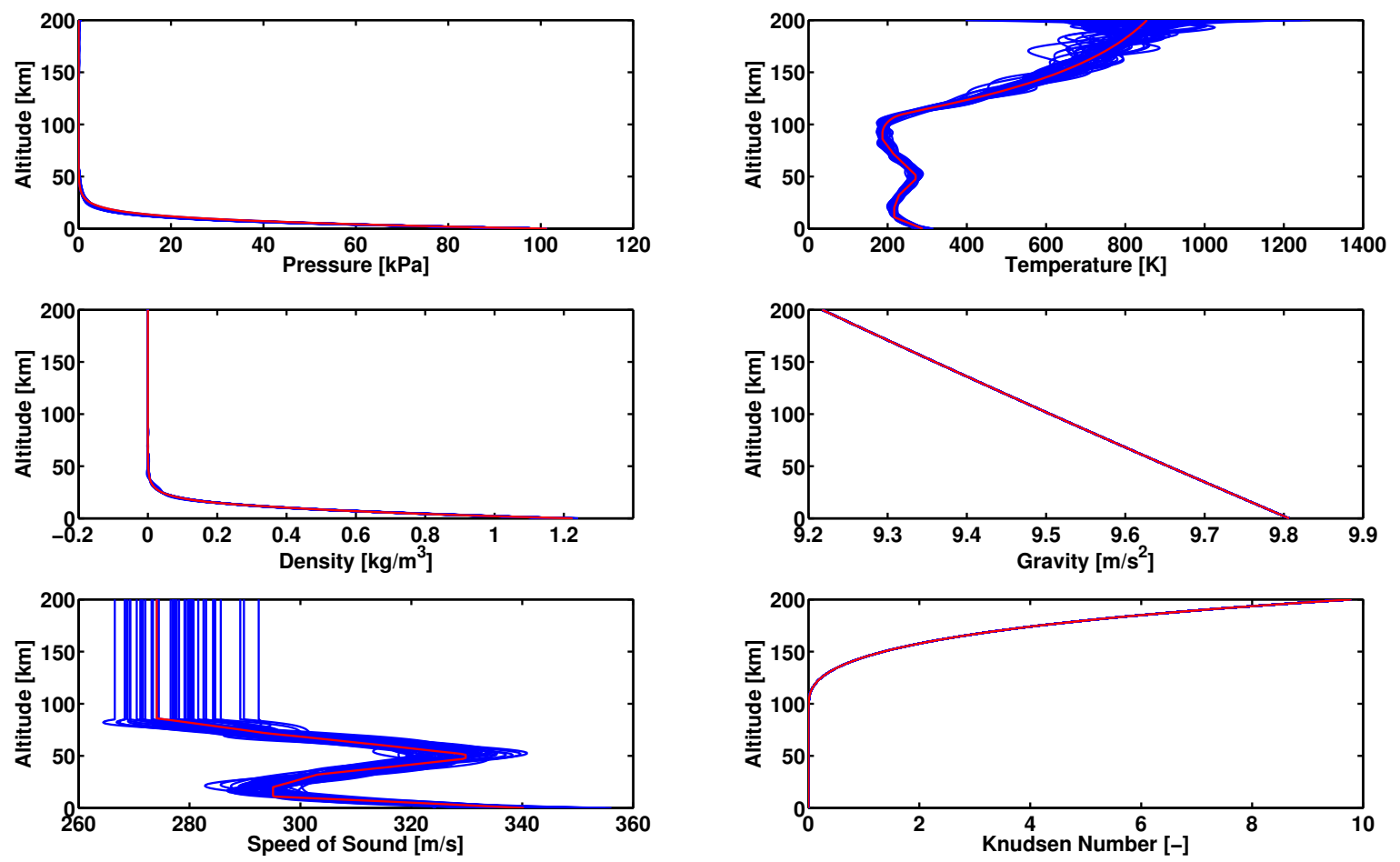

Figure 4: 1976 US Standard Atmosphere subject to uncertainty in the temperature variation with altitude (nominal atmospheric conditions in red). The uncertainty in the atmospheric temperature is taken to vary from $10 \%$ of the nominal value (at sea level) to $30 \%$ at $200 \mathrm{~km}$ altitude.

\section{Trajectory Optimisation}

7 HIS section presents the various mathematical models that are used in conjunction with the aero-thermal 1 model within HyFlow to simulate the performance of our representative RLV during its un-powered reentry into the Earth's atmosphere.

\section{V.A. Earth model}

The gravitational acceleration $g$ is assumed to vary with altitude according to an inverse square law

$$
g(h)=g_{0}\left(\frac{h}{R_{E}+h}\right)^{2}
$$

where $h$ denotes the altitude above mean sea level, $R_{E}=6375 \mathrm{~km}$ is the mean radius of the Earth, and $g_{0}=$ $9.80665 \mathrm{~m} / \mathrm{s}^{2}$ is the gravitational acceleration at sea level. The atmospheric characteristics (temperature, pressure, density and speed of sound) follow the 1976 US Standard Atmosphere model up to $1000 \mathrm{~km}$. The effects of wind are not accounted for. 


\section{V.B. Dynamic model}

The vehicle is represented dynamically by a simple point mass with added rigid body characteristics (see section V.C on the following page). The translational motion of the vehicle along its trajectory is governed by the following set of differential equations: ${ }^{17}$

$$
\begin{aligned}
\dot{h}= & v \sin \gamma \\
\dot{v}= & -\frac{D}{m}-g \sin \gamma+\omega_{E}^{2}\left(R_{E}+h\right) \cos \lambda(\sin \gamma \cos \lambda-\cos \gamma \sin \chi \sin \lambda) \\
\dot{\gamma}= & \frac{L}{m v} \cos \mu-\left(\frac{g}{v}-\frac{v}{R_{E}+h}\right) \cos \gamma+2 \omega_{E} \cos \chi \cos \lambda \\
& \quad+\omega_{E}^{2}\left(\frac{R_{E}+h}{v}\right) \cos \lambda(\sin \chi \sin \gamma \sin \lambda+\cos \gamma \cos \lambda) \\
\dot{\chi}= & \frac{L}{m v \cos \gamma} \sin \mu-\left(\frac{v}{R_{E}+h}\right) \cos \gamma \cos \chi \tan \lambda \\
\dot{\lambda}= & \left(\frac{v}{R_{E}+h}\right) \cos \gamma \sin \chi \\
\dot{\theta}_{L}= & \left(\frac{v}{R_{E}+h}\right) \frac{\cos \gamma \cos \chi}{\cos \lambda}
\end{aligned}
$$

where $v$ is the speed of the vehicle as measured in an Earth-centred reference frame (assumed to have a rotation rate $\omega_{E}=7.2921 \times 10^{-5} \mathrm{rad} / \mathrm{s}$ ). In these equations, $\gamma$ indicates the flight path angle, $\chi$ is the path directional angle, $\mu$ is the bank angle, and $\lambda$ and $\theta_{L}$ denote respectively the latitude and the longitude of the vehicle at time $t$. $L$ and $D$ denote the aerodynamic lift and drag forces, obtained by resolving $\hat{F}$, given by Eq. 8 on page 5 , into its components in wind axes. The mass of the vehicle, $m$, is assumed to be constant at $60,000 \mathrm{~kg}$ throughout the re-entry trajectory. A control law governs the angle of attack $\alpha$ and the bank angle $\mu$, allowing the vehicle to perform out-of-plane motions during its descent.

\section{V.C. Controls model}

The controls of our representative RLV are comprised of two fins arranged in a butterfly configuration at the rear of the fuselage, which are used both for yaw and roll control, as well as for pitch control in low-speed flight. The rear fuselage, flattened into a pen-nib shape, accommodates a rear beaver-tail control surface a la Space Shuttle for pitch attitude control during reentry. Finally, two elevons are attached to the trailing edge of the wings for additional pitch and roll control. The vehicle, together with its control surfaces, is depicted in Fig. 5 on the following page.

The deflections of the controls are obtained by accounting for the rotational motion of the vehicle as follows. Firstly the components $\mathrm{p}, \mathrm{q}$ and $\mathrm{r}$ in body axes of the rate of rotation of the vehicle about its centre of mass can be related to the rates of change of the pitch angle $\theta$ (i.e. $=\alpha+\gamma$ ), bank angle $\mu$, and heading angle $\chi$ at any point along the trajectory via the Euler angle transformation

$$
\left[\begin{array}{l}
p \\
q \\
r
\end{array}\right]=\left[\begin{array}{c}
\frac{\partial \mu}{\partial t}-\frac{\partial \chi}{d t} \times \sin \theta \\
\frac{\partial \theta}{\partial t} \times \cos \mu+\frac{\partial \chi}{\partial t} \cos \theta \sin \mu \\
-\frac{\partial \theta}{\partial t} \times \sin \mu+\frac{\partial \chi}{\partial t} \times \cos \theta \cos \mu
\end{array}\right]
$$

These components can then be differentiated (numerically or otherwise) with respect to time to obtain the angular accelerations $\dot{p}, \dot{q}$ and $\dot{r}$ of the vehicle about its centre of mass (again in body axes). These accelerations can then be related via Euler's law to the body-axis components $M_{x}, M_{y}$ and $M_{z}$ of the aerodynamic moment $\hat{M}$ (see Eq. 9 on page 5) required to generate these accelerations:

$$
\left[\begin{array}{c}
M_{x} \\
M_{y} \\
M_{z}
\end{array}\right]=I_{v}^{B} \times\left[\begin{array}{c}
\dot{q} \\
\dot{r} \\
\dot{p}
\end{array}\right]
$$


where the matrix $I_{v}^{B}$ contains the body-axis components of the inertia tensor of the vehicle. The set of control surface deflections $\left\{\delta_{i}\right\}$ required to produce these moments can then be obtained as described in section VI.B on the following page. In our present approach, the aerodynamic effects of changing the control surface deflections are obtained directly from Hyflow by applying the appropriate three-dimensional affine transformations to the mesh defining the surface of the vehicle in order to rotate the control surfaces.

Based on the assumed mass of the vehicle and a uniform distribution of its structural density, the components of $I_{v}^{B}$ for our representative vehicle were assumed as follows:

$$
I_{v}^{B}=\left[\begin{array}{ccc}
I_{x x} & I_{x y} & I_{x z} \\
I_{y x} & I_{y y} & I_{y z} \\
I_{z x} & I_{z y} & I_{z z}
\end{array}\right]=\left[\begin{array}{ccc}
7.876 \times 10^{5} & 0.117 & -0.072 \\
0.117 & 8.580 \times 10^{5} & -2.603 \times 10^{4} \\
-0.072 & -2.603 \times 10^{4} & 8.674 \times 10^{4}
\end{array}\right]\left[\mathrm{kg} / \mathrm{m}^{2}\right]
$$

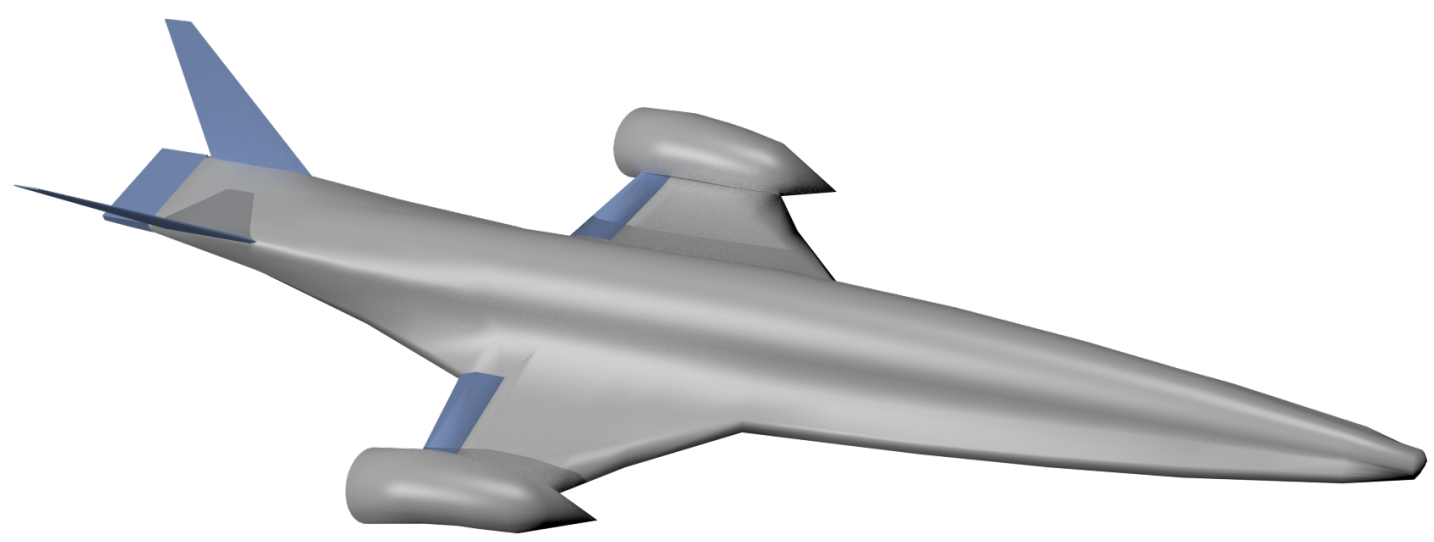

Figure 5: CFASTT-1 vehicle with its control surfaces.

\section{Robustness Analysis}

$\mathrm{T}$ HE characterisation of the sensitivity of the design of our representative RLV to the effects of roughnessinduced transition, as exacerbated by uncertainty in the atmospheric conditions, can be phrased as an optimal control problem and divided into three distinct steps:

1. The optimal design of a nominal trajectory for our representative RLV where the effect of roughnessinduced boundary layer transition is neglected and nominal values are assumed for the atmosphere through which the vehicle passes,

2. The calculation of the perturbation to the nominal trajectory that results from the presence of roughness-induced transition and uncertainty in the atmospheric conditions pertaining along the flightpath, and

3. The computation of the optimal scheduling of control surface deflections that enables the vehicle to fly the desired nominal trajectory even in the presence of roughness-induced transition and uncertainty in the atmospheric conditions.

A statistical picture of the robustness of the design can then be built up by performing a Monte Carlo analysis in which elements (2) and (3) above are repeated a large number of times, each time sampling both the parameters governing the variation of atmospheric properties with altitude and those that govern the position and character of the onset of roughness-induced transition from their appropriate statistical distributions. 


\section{VI.A. Optimisation Methodology}

The selection of an appropriate set of optimisation tools for the design of vehicle trajectories is never a particularly straightforward process. The convergence of conventional, simple gradient-based optimisation routines is often severely hampered by particular features that are embedded within the model for the system's performance. Changes in behavioral mode or other discontinuities embedded within the model cause particular problems in this respect. The choice of optimisation algorithm can thus influence the outcome of the design process considerably, and it can be difficult, if not impossible, to find the true, globally-optimal design solution even if a good initial guess is available. The method used in the present work is based on a mixed approach. A population-based stochastic algorithm is used first to explore the design space and to identify a set of neighbourhoods in which candidates for the global optimum of the system might exist. A gradient-based approach is then used in each such neighbourhood to refine the solution and to ensure that the constraints on the system are accurately met. ${ }^{18}$ Following this, the highest ranked solution according to its cost function is accepted as the global optimum. To optimise the re-entry trajectory of our representative RLV, a direct collocation method based on the Finite Elements in Time (FET) approach and using a spectral basis ${ }^{19}$ is applied: in this approach, the trajectory is discretised into a large but finite number $N$ of subintervals, and the design space is then the matrix product of the unit vector of dimension $N$ and a suitable control vector $c$ for the vehicle along its trajectory. The resultant non-linear programming (NLP) problem ${ }^{20}$ is then solved using the MOPED ${ }^{21}$ algorithm to search for candidate optima; these solutions are then refined using the gradient-based IDEA ${ }^{22}$ optimisation method.

\section{VI.B. Trajectory Integration}

To obtain the re-entry trajectory for our representative RLV, the control vector $c$ is defined as

$$
c=\left[\alpha, \mu, t_{f}\right]
$$

where $t_{f}$ is the duration of the trajectory from initiation until the vehicle reaches its TAEM interface. The search space $D$ for the controls is defined by the bounds $\alpha \in\left[-10^{\circ}, 75^{\circ}\right], \mu \in\left[-85^{\circ}, 85^{\circ}\right]$, and the flight time is set not to exceed 3000 seconds. The control law $c(t)$ is then obtained as the solution of the optimisation problem which aims to minimize the integrated heat load at the nose stagnation point:

$$
\min _{\mathbf{c} \in D} \int_{t_{0}}^{t_{f}} \dot{q}_{s t}(t) d t
$$

subject to the dynamics described in the previous section and initial conditions (i.e. at $t=0$ ) set to start in the hypersonic regime: $h_{0}=120 \mathrm{~km}, v_{0}=7.8 \mathrm{~km} / \mathrm{s}, \gamma_{0}=-1^{\circ}, \chi_{0}=90^{\circ}, \lambda_{0}=1^{\circ}, \theta_{L 0}=0^{\circ}$. The terminal conditions (i.e. at $t=t_{f}$ ) are $h_{f}=24 \mathrm{~km}, v_{f}=0.8 \mathrm{~km} / \mathrm{s}, \gamma_{f}=-30^{\circ}, \chi_{f}=90^{\circ}, \lambda_{f}=40^{\circ}$, and $\theta_{L f}=0^{\circ}$. Additional path constraints are imposed on the maximum acceleration along the normal body axis so that $a_{y}(t) \leq 28 \mathrm{~m} / \mathrm{s}^{2}$ and the peak heat flux at the nose stagnation point $\dot{q}_{s t}(t)<750,000 \mathrm{~W} / \mathrm{m}^{2}$.

\section{Controls Scheduling}

In order to determine the variation of control surface deflection that is required along the re-entry trajectory, the control vector $c_{\delta}$ is first defined as

$$
c_{\delta}=\left[\delta_{1}, \delta_{2}, \delta_{3}, \delta_{4}, \delta_{5}\right]
$$

where $\delta_{1}$ and $\delta_{2}$ correspond to the rotation angles of the rear fins, $\delta_{3}$ and $\delta_{4}$ correspond to the rotation angles of the elevons, and $\delta_{5}$ controls the rotation angle of the beaver tail (see section V.C on page 10). The search space $\mathrm{D}$ for the control deflection angles $\delta_{i}$ is defined by the bounds $\delta_{1,2} \in\left[-30^{\circ}, 30^{\circ}\right], \delta_{3,4} \in\left[-10^{\circ}, 40^{\circ}\right]$, and $\delta_{5} \in\left[-20^{\circ}, 40^{\circ}\right]$. The optimal scheduling of the control surface deflections, $c_{\delta}(t)$, is then defined as the solution to the multi-objective pointwise optimisation problem which aims to minimize both the effects of asymmetric transition (Eq. 29) and the aerodynamic penalties (Eq. 30) induced by attitude correction, while maintaining the desired attitude of the reentry vehicle:

$$
\begin{gathered}
\min _{\mathbf{c}_{\delta} \in D}\left\|\hat{M}_{\text {controls }}-\hat{M}_{\text {nom }}\right\| \\
\min _{\mathbf{c}_{\delta} \in D}\left\|P_{\text {nom }}-P\right\|
\end{gathered}
$$


where $\hat{M}_{n o m}$ is the aerodynamic moment that is required to generate the rotational dynamics of the vehicle that are consistent with its desired nominal trajectory (Eq. 24 on page 10) and $\hat{M}_{\text {controls }}$ is the aerodynamic moment that is generated by the vehicle with the control surfaces deflected. Similarly $P_{\text {nom }}=(L / D)_{\text {nom }}$ and $P=(L / D)$ are, respectively, representative measures of the aerodynamic performance of the vehicle under nominal conditions, and where deviations from nominal conditions along the trajectory (for example, due to perturbations in the atmospheric conditions or variability in the aerodynamic loads as a result of uncertainties in the position and time of boundary layer transition) might possibly require the controls to be deflected by an additional amount. This second, "disturbance rejection", element to the optimisation in effect biases the scheduling of the control surfaces towards a solution that, even in the presence of aerodynamic perturbations, keeps the trajectory of the vehicle as close as possible to its nominal optimal trajectory.
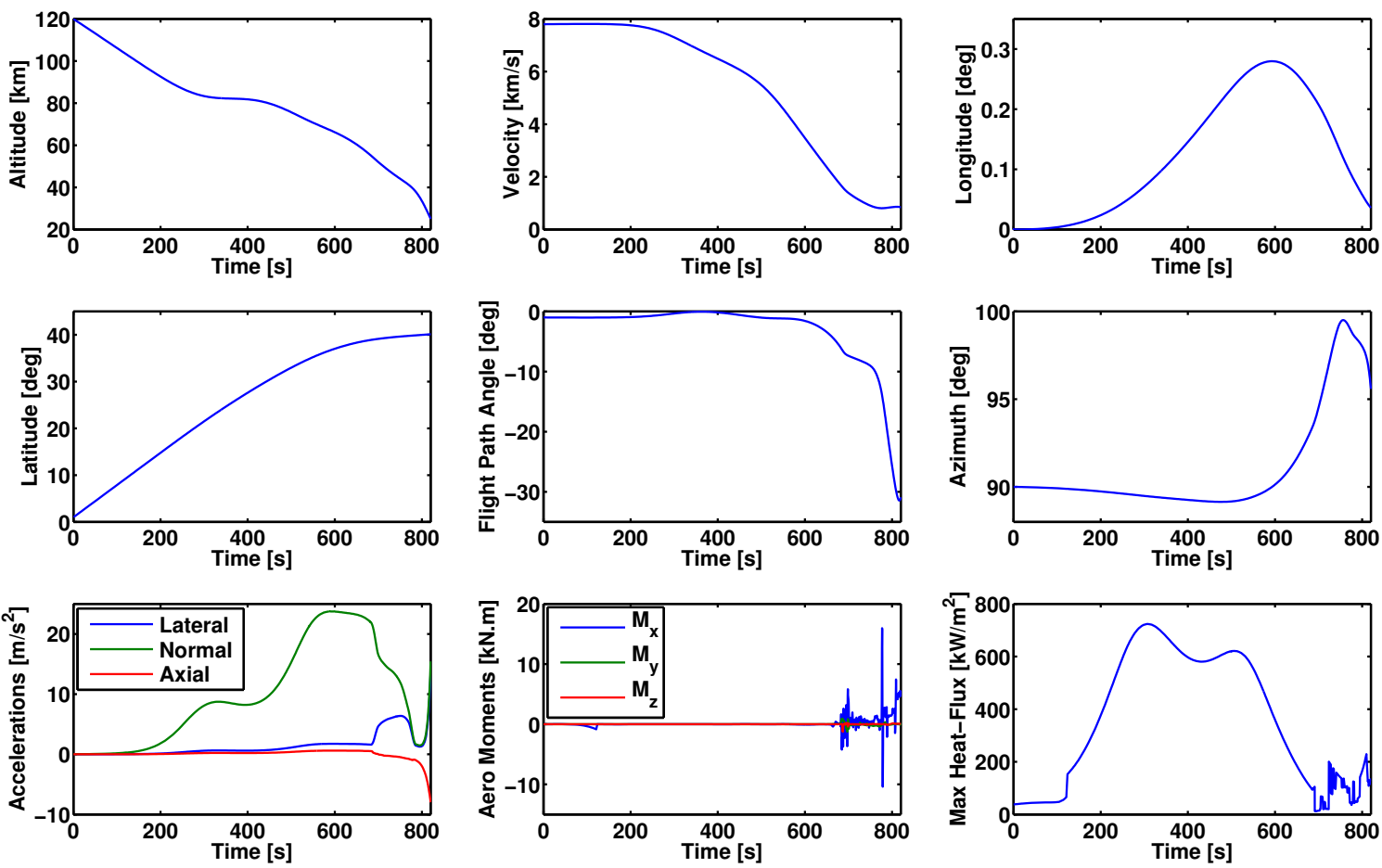

Figure 6: Nominal re-entry trajectory of the CFASTT-1 vehicle.

\section{VI.C. Nominal Trajectory}

The resultant nominal trajectory of the vehicle, as generated using the procedure outlined in Section VI.B on the previous page, is shown in Fig. 6. It should be remarked at the outset that the geometric shape of the CFASTT-1 vehicle that is the subject of the present study (see Fig. 1 on page 3) was specifically optimised, through its use of nose-strakes and a sculpted rear fuselage, to adopt a natural nose-high attitude during re-entry and thus to minimise the intervention required from the control system in comparison to some other vehicles with similar configuration. The shape optimisation procedure that allowed this to be done is beyond the scope of the present work, however, and will be discussed in detail in a later paper.

During the first phase of the re-entry, the vehicle maintains its descent at a constant $75^{\circ}$ angle of attack (see Fig. 7 on the following page) as a means of decelerating within the upper, less dense, regions of the terrestrial atmosphere. The vehicle is subjected to the maximum aerodynamic heating at its nose stagnation region shortly after crossing the mesopause at about $80 \mathrm{~km}$ altitude. The scheduled angle of attack is then promptly reduced in order to guarantee the structural integrity of the vehicle as its normal acceleration increases towards the design threshold (set to $28 \mathrm{~m} / \mathrm{s}^{2}$ ) at about 600 seconds after the beginning of the re-entry phase. A final set of combined pitch/roll inputs are required to turn the vehicle onto its correct 
heading during the terminal manoeuvres that bring it to the prescribed final latitude and longitude of the TAEM interface.

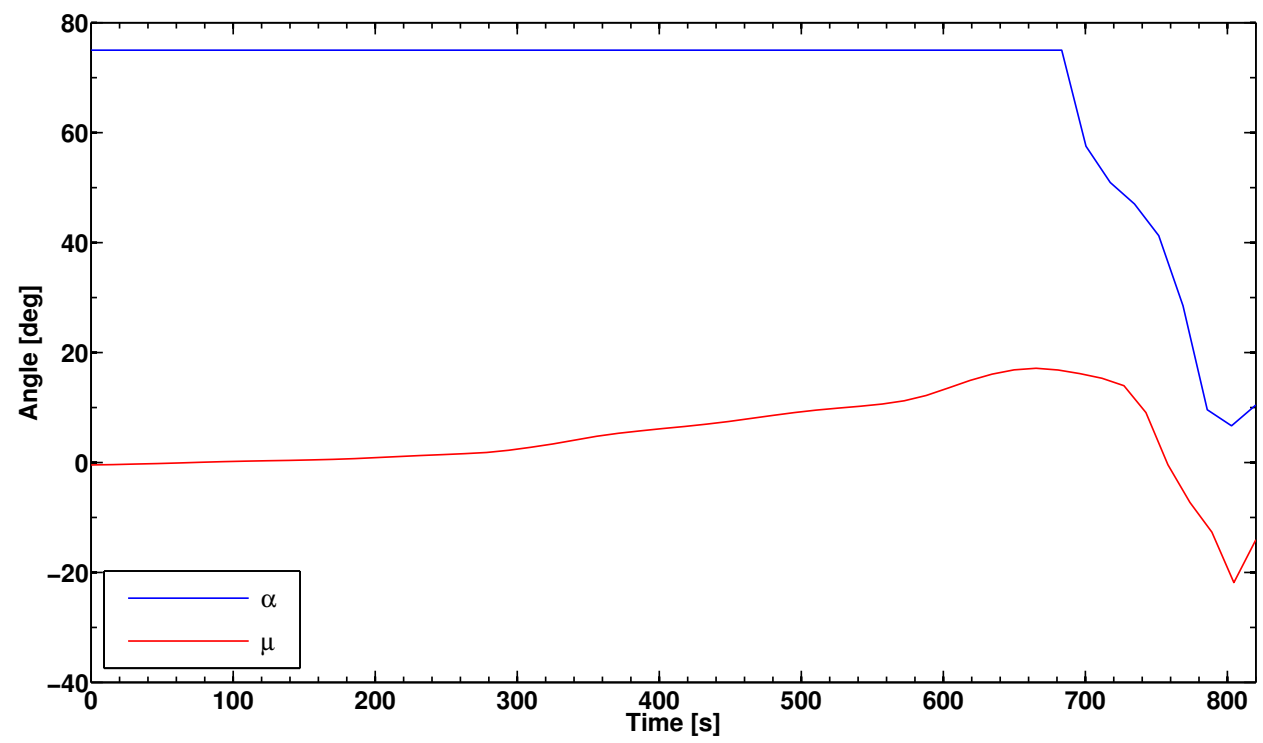

Figure 7: Nominal schedule of bank angle $\mu$ and angle of attack $\alpha$.

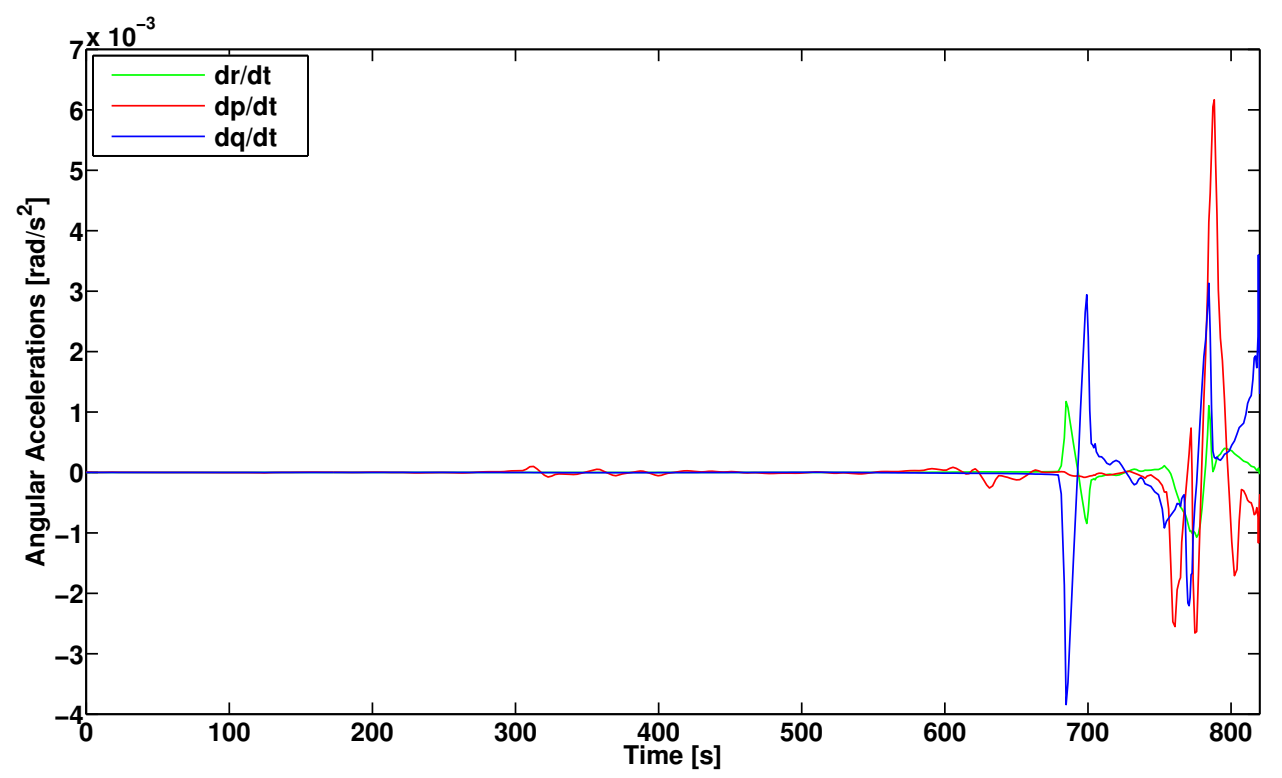

Figure 8: Angular accelerations in pitch $(\dot{q})$, yaw $(\dot{r})$, and roll $(\dot{p})$ along the vehicle's nominal trajectory.

\section{Controls Scheduling for the Nominal Trajectory}

The variation along the nominal trajectory of the components $\dot{p}, \dot{q}$ and $\dot{r}$ in body axes of the angular acceleration of the vehicle about its centre of mass is shown in Fig. 8. Following the procedure summarized in Section V.C on page 10, the moments that need to be generated in order to rotate the vehicle into the desired attitude can then be obtained from Eq. 24 on page 10. The variation of these moments along the nominal trajectory of the vehicle are shown in Fig. 9 on the following page. The optimal scheduling of the control surface deflections is then determined at each of the collocation points along the trajectory that are also shown in this figure. In the first phase of the trajectory, during which the vehicle operates at approximately constant angle of attack and bank angle, a low density of collocation points is adequate to 
capture accurately the required variations in control surface deflection, whereas from $t=664 s$ onwards, the attitude of the vehicle changes rapidly enough for a far denser distribution of collocation points to be required.

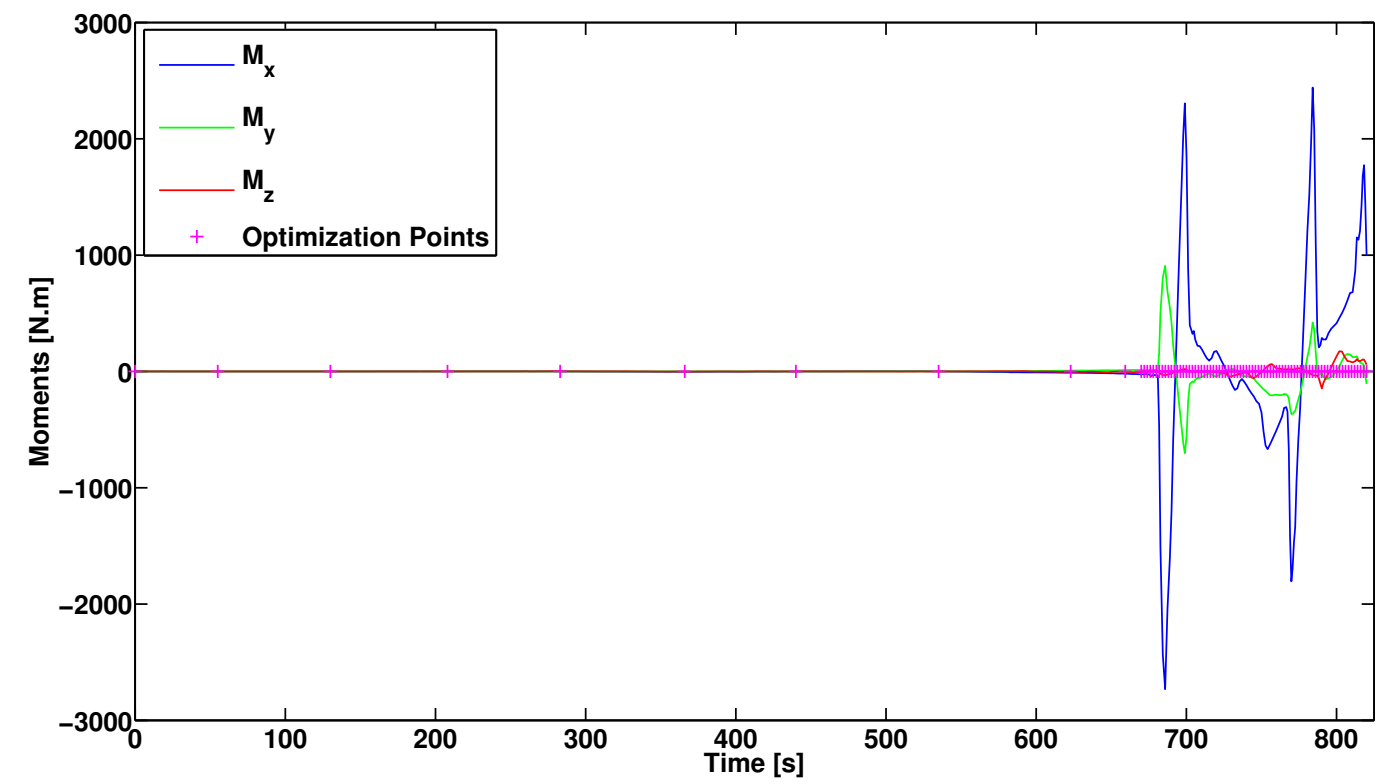

Figure 9: Aerodynamic moments required to generate the nominal angular accelerations of the vehicle: pitch moment $M_{x}$, yaw moment $M_{y}$, roll moment $M_{z}$.

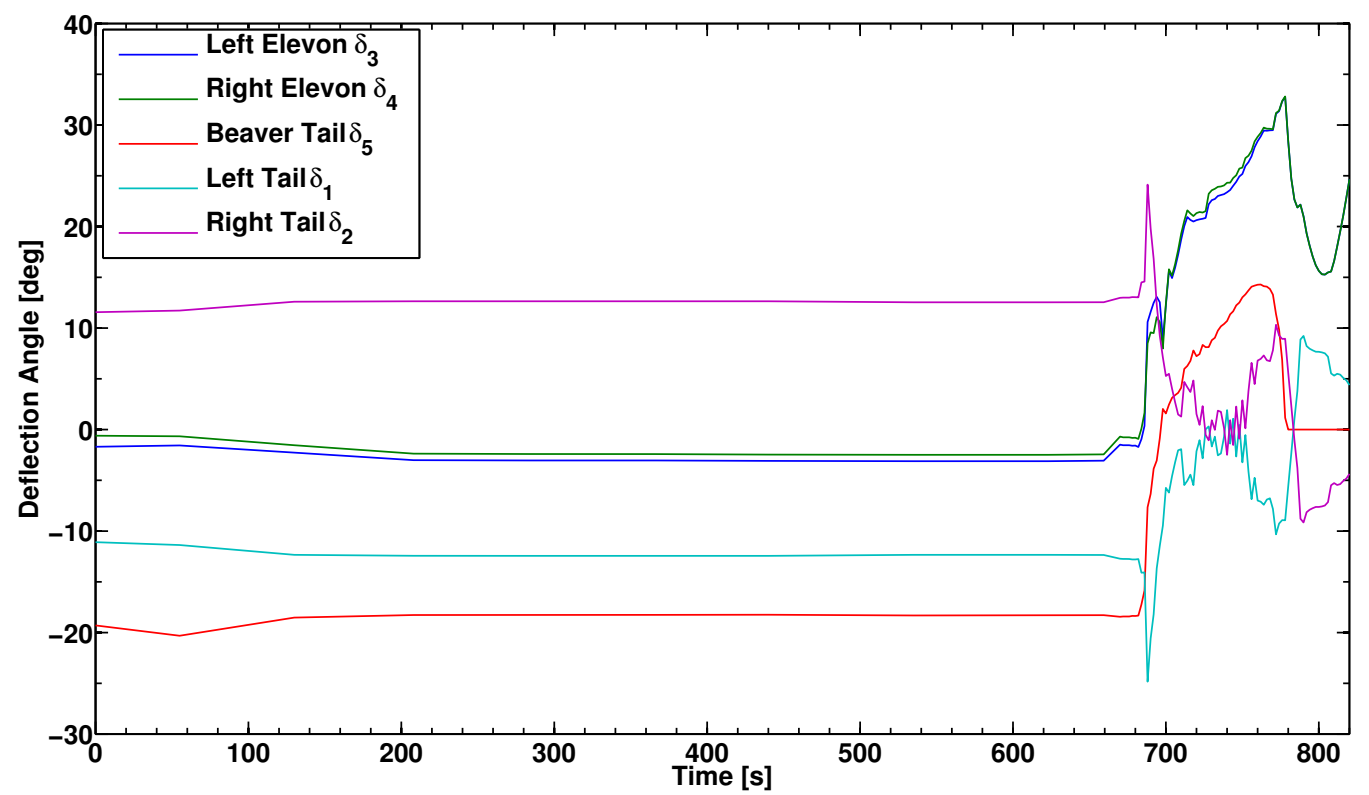

Figure 10: Deflection of the control surfaces along the nominal re-entry trajectory of the CFASTT-1 vehicle.

The deflections of the control surfaces along the nominal trajectory of the CFASTT-1 vehicle, obtained via the methodology outlined in section VI.B on page 12, are presented in Fig. 10. If the very low-amplitude fluctuation in the control surface deflections is disregarded, all of the control surfaces can be seen to maintain an almost steady deflection during the preliminary phase of the re-entry at very high angle of attack, vindicating the minimal-control aerodynamic design philosophy that was used to generate the shape of the vehicle as alluded to in section VI.C on page 13. As was intended, the beaver tail is the control surface which participates most in the control of the vehicle throughout this preliminary phase of the trajectory by 
maintaining the pitch attitude of the vehicle. At the onset of the manoeuvre which leads to a rapid decrease in the pitch attitude of the vehicle, the elevons and rear fins begin to take over the attitude control of the vehicle. Indeed, a point is reached, at approximately $780 \mathrm{~s}$ into the trajectory, at which the beaver tail is aerodynamically shadowed by the forward fuselage of the vehicle, and is commanded therefore to return to its neutral attitude. The absolute error between the required moment and the moment generated by the vehicle at each optimization point along its nominal trajectory is presented in Fig. 11. The relatively small deviations throughout give confidence in the ability of the current control scheme to manage the changes in the vehicle's attitude that are required in order for it to achieve its nominal trajectory.

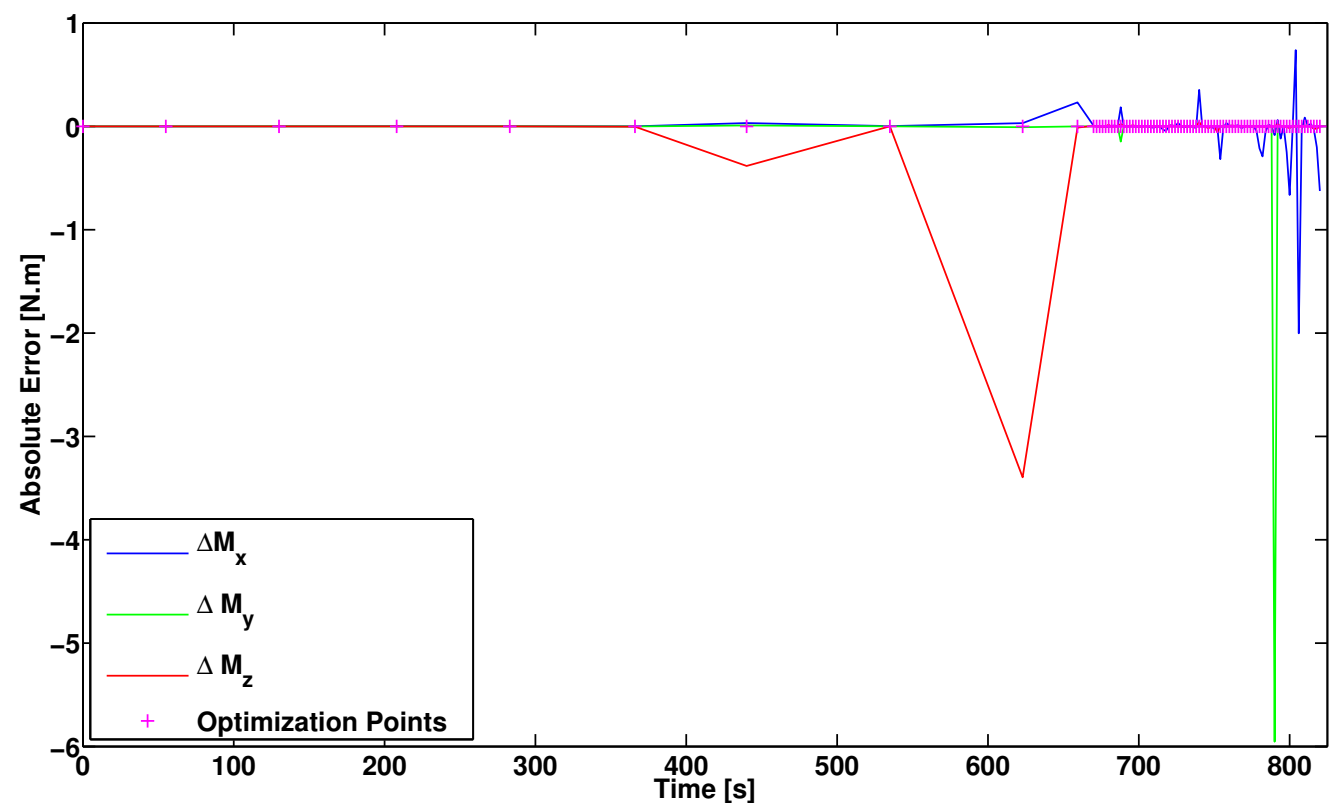

Figure 11: Absolute error between the required moment and the moment generated by the vehicle along its nominal trajectory: pitch moment $\Delta M_{x}$, yaw moment $\Delta M_{y}$, roll moment $\Delta M_{z}$.

\section{VI.D. Smooth-surface Transition}

A Monte-Carlo simulation consisting of a hundred different re-integrations of the reentry trajectory with the nominal control law and randomizing the atmospheric temperature profile (see Section IV.B.2 on page 7) was performed to examine the effect of uncertainty in the atmospheric conditions pertaining during re-entry on the altitude at which smooth-surface laminar-turbulent transition takes place on the surface of the vehicle, thus on its aerodynamic performance and subsequently on its ability to satisfy its mission objectives. Bearing in mind the various assumptions within the models, the effect of uncertainty in the atmospheric temperature appears to be to create a quasi-Gaussian distribution of transition altitudes about the nominal height for transition (predicted here to be about $57.3 \mathrm{~km}$ ) with a slight bias towards transition taking place at a higher than nominal altitude (the distribution mean is predicted to be about $57.5 \mathrm{~km}$ and the most likely value about $57.7 \mathrm{~km}$, bearing in mind the limitations of the relatively small sample size in identifying these values accurately). Since smooth-surface boundary layer transition occurs at such low altitudes on a vehicle with this configuration, its effect (in isolation) on the heating rates experienced by the vehicle are minimal (see Fig. 6 on page 13 - bottom right). The resultant effect on the trajectory of the vehicle is shown in Fig. 13 on the next page, where the distribution of final longitudes and latitudes that result in the presence of variability in the atmospheric conditions is plotted. These figures suggest the relatively small effect of atmospheric variability and smooth-surface transition on the aerodynamics of this particular vehicle: in the presence of these effects, the end point of its re-entry trajectory exhibits a scatter of only about $0.5^{\circ}$ in final latitude and $0.01^{\circ}$ in final longitude (equivalent to a scatter of about 55 and $1 \mathrm{~km}$ respectively in position on the surface of the Earth). 


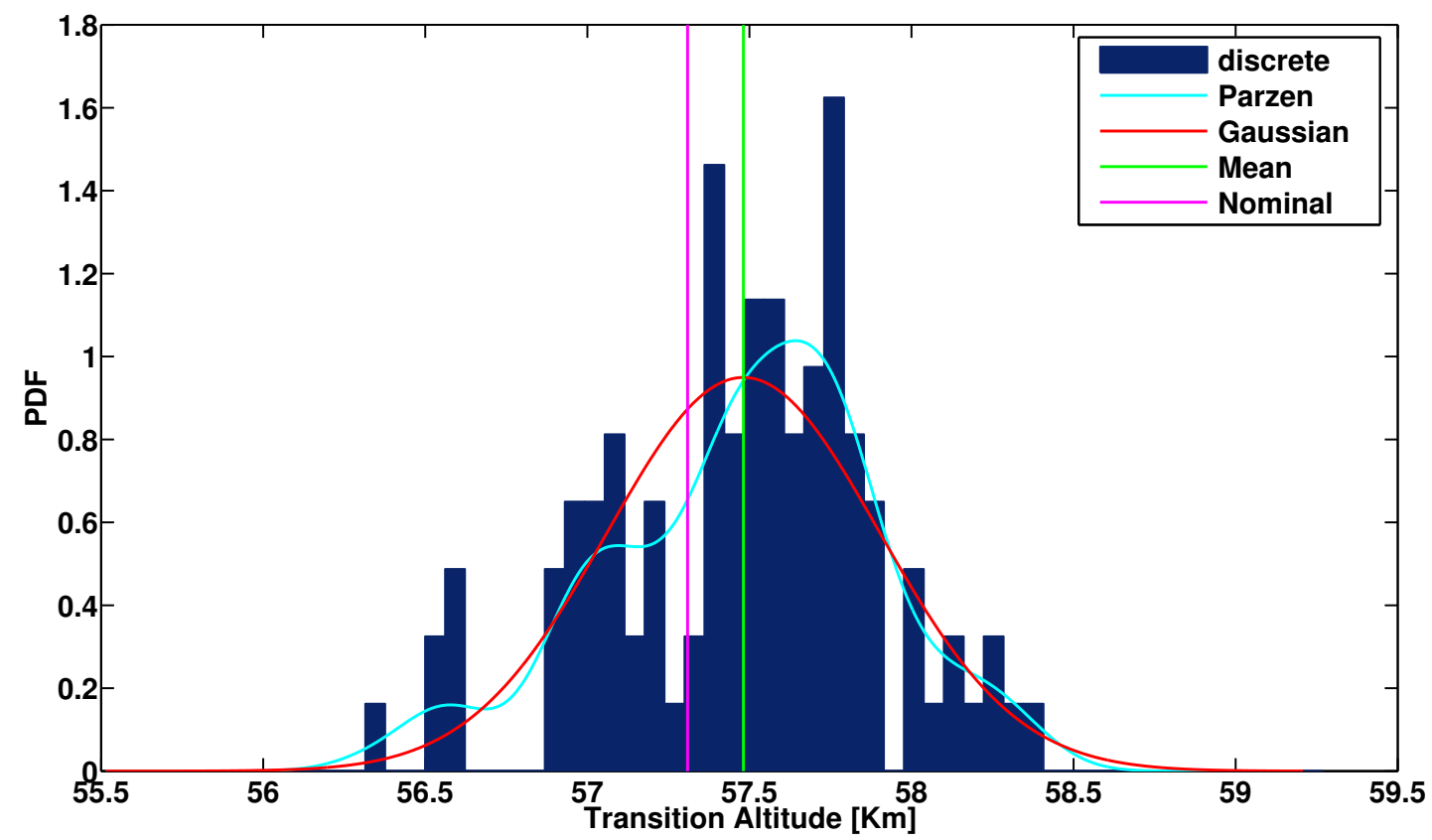

Figure 12: Result of a Monte-Carlo simulation with 100 sample reentry trajectories to estimate the effect of variability within the atmospheric temperature profile on the altitude at which smooth-surface transition of the boundary layer on the surface of the CFASTT-1 vehicle is triggered.
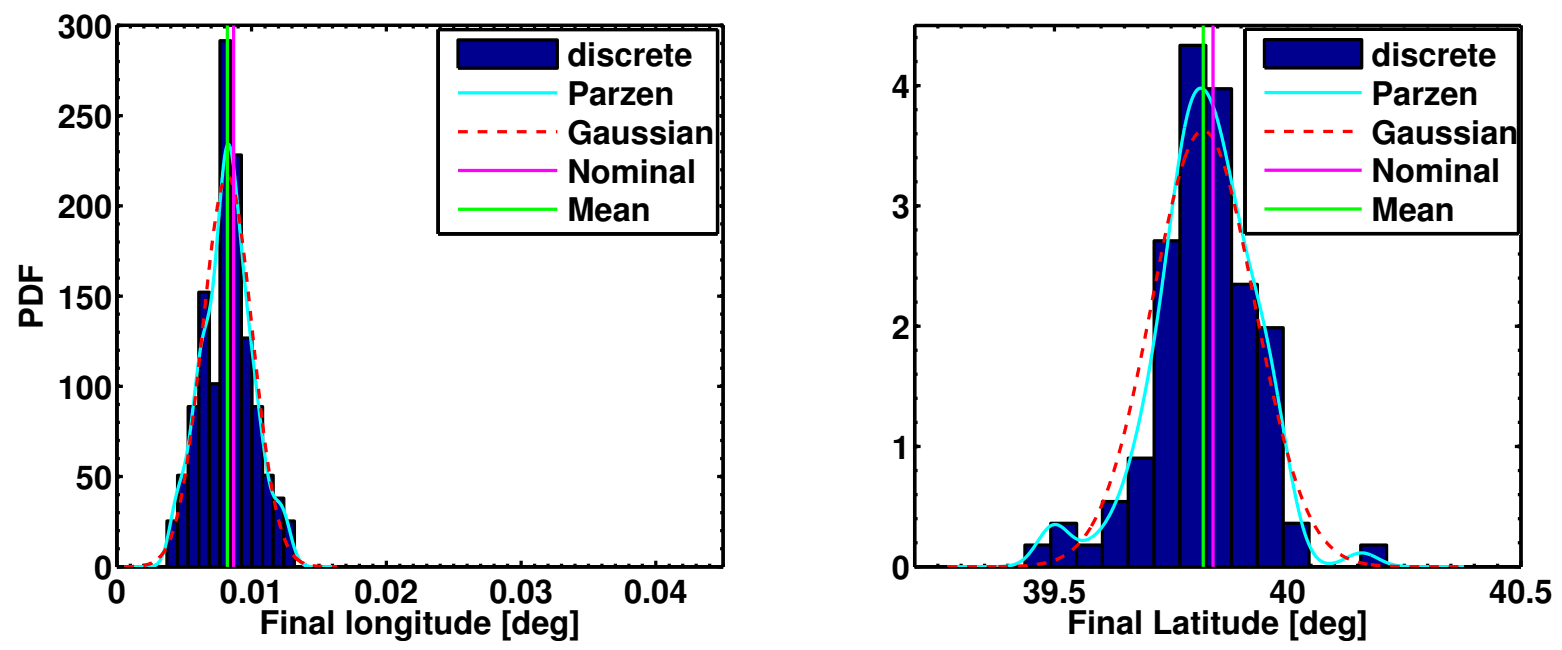

Figure 13: Result of a Monte-Carlo simulation with 100 sample reentry trajectories to estimate the effect of variability within the atmospheric temperature profile on the altitude at which smooth-surface transition of the boundary layer on the surface of the CFASTT-1 vehicle is triggered, showing the resultant spread of final longitudes and latitudes about the nominal.

\section{VI.E. Rougness-Induced Transition}

Additional variability is introduced into the model if, in addition to smooth-surface transition, transition from laminar to turbulent flow can also take place through the formation of turbulent wedges on the surface of the vehicle. This can happen as the result of the presence of localised elements of roughness on the surface of the vehicle, as described in section IV.B.1 on page 6 . In the present study, a set of 20 distinct trajectories were generated with an element of surface roughness located at various different positions on 


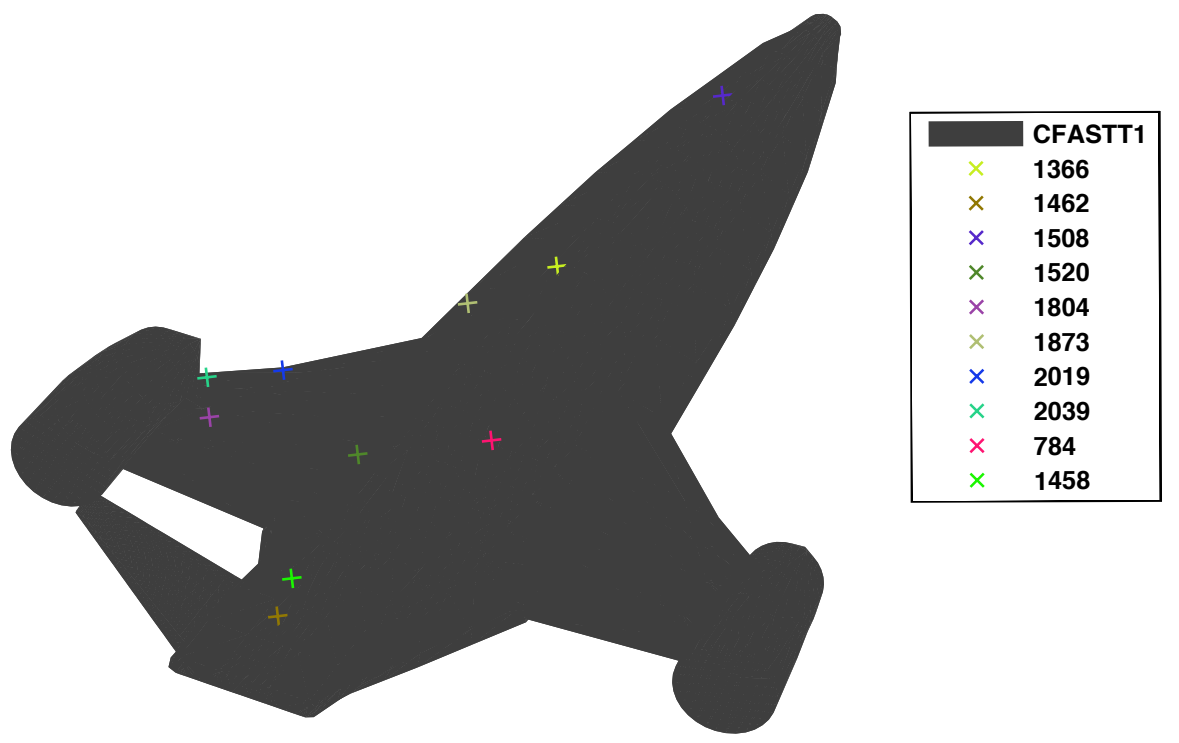

Figure 14: The locations of the various simulated surface asperities used to trigger the onset of roughnessinduced laminar-turbulent transition.

the right side of the under-surface of the vehicle as depicted in Fig. 14 on the next page. Calculations were repeated for elements with height $h_{1}=4 \mathrm{~cm}$ and $h_{2}=6 \mathrm{~cm}$ to examine the sensitivity of predictions to the size of the surface asperities that might be responsible for transition. The effect of the resultant turbulent wedge regions on the controllability of the vehicle was evaluated in terms of the perturbations from nominal, denoted $\Delta \hat{M}$, that the presence of the turbulent wedge induced in the aerodynamic moments acting on the vehicle. The resultant evolution along the trajectory of the perturbations about the pitch, yaw and roll axes, for the various roughness locations that were considered, is presented in Fig. 15 on the following page. As can be seen, the direct effect of the presence of roughness-induced transition is to introduce an additional contribution to the shear force on the surface of the vehicle, which, if asymmetrically located with respect to the centre of mass, temporarily unbalances the aerodynamic forces acting on the vehicle and must be counteracted by an additional deflection of the control surfaces away from their nominal positions. The influence of the height of the roughness element, $h_{\text {wedge }}$, is to modify the time at which the onset of transition of the boundary layer takes place. Indeed, for a roughness element with height $h_{1}=4 \mathrm{~cm}$, the earliest time of occurence of transition is predicted to be about $635 \mathrm{~s}$ (i.e. with the vehicle at an altitude of about $60 \mathrm{~km}$ - compared to the $57 \mathrm{~km}$ or so at which smooth-surface transition takes place), while if the characteristic height of the local surface asperity is increased to $6 \mathrm{~cm}$, the formation of turbulent wedges can occur somewhat earlier (at about $610 \mathrm{~s}$ into the descent, with the vehicle at an altitude of about $65 \mathrm{~km}$ ). This behaviour is fully consistent with experience on vehicles such as the Shuttle.

As expected, the presence of a roughness element located close to the centreline, e.g. element 784 in Fig. 14, has very little influence on the overall aerodynamic characteristics of the vehicle, while, as can be seen in Fig. 15 on the following page, if the roughness is located slightly further off the centreline, e.g. elements 2019 and 1508 in Fig. 14, the region of turbulent flow within the resultant turbulent wedge can be responsible for a significant increase in moment about the centre of mass of the vehicle. As the vehicle descends, the wedge continues to develop, its apex angle changing with Mach number according to Eq. 12 on page 7. The resultant steady increase in the effects of the turbulent wedge, as the vehicle decelerates, is clearly visible in the trend of the data, as is the point at which the the smooth-surface turbulent region starts to subsume the turbulence in the wedge and hence the loads on the vehicle start to return towards their nominal values. The growth of the smooth-surface turbulent region is not fast enough in all cases, however, 

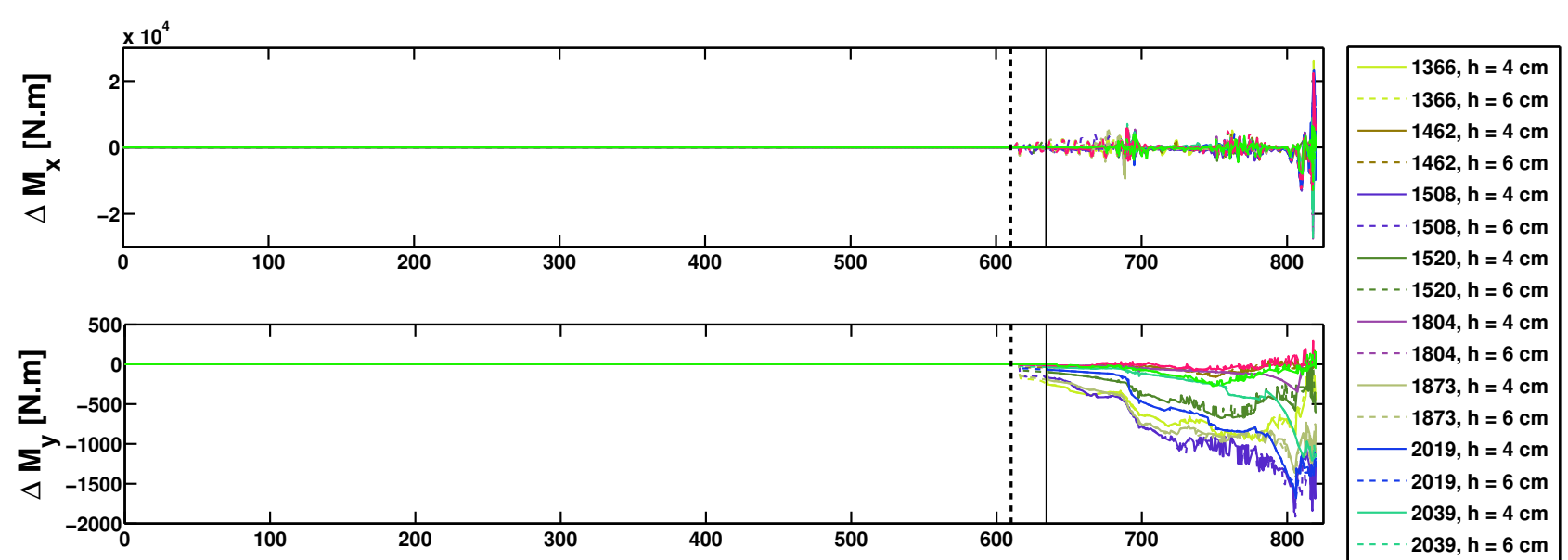

$1804, \mathrm{~h}=4 \mathrm{~cm}$

$1804, \mathrm{~h}=6 \mathrm{~cm}$

1873, $h=4 \mathrm{~cm}$

$1873, \mathrm{~h}=6 \mathrm{~cm}$

2019, $\mathrm{h}=4 \mathrm{~cm}$

- 2019, $h=6 \mathrm{~cm}$

2039, $h=4 \mathrm{~cm}$

2039, $h=6 \mathrm{~cm}$

-784, $h=4 \mathrm{~cm}$

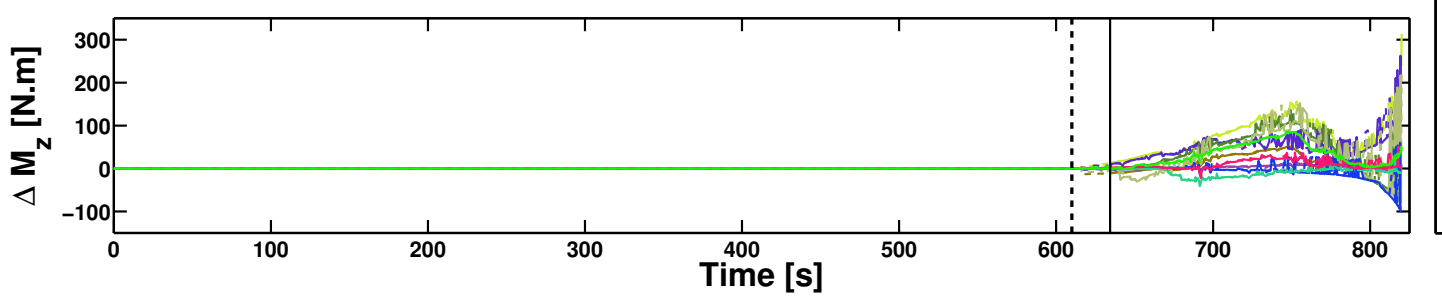

- 784, $h=6 \mathrm{~cm}$

$1458, \mathrm{~h}=4 \mathrm{~cm}$

$---1458, h=6 \mathrm{~cm}$

$\ldots . t_{\text {trip }}, h=6 \mathrm{~cm}$

$\longrightarrow t_{\text {trip }}, h=4 \mathrm{~cm}$

Figure 15: Perturbations to the nominal aerodynamic moments in the presence of roughness-induced boundary layer transition on the surface of the vehicle: pitch moment $\Delta M_{x}$, yaw moment $\Delta M_{y}$, roll moment $\Delta M_{z}$.

to fully subsume the effects of the turbulent wedges by the time the vehicle reaches the terminal point of its trajectory. Indeed, at the TAEM interface only about $73 \%$ of the flow over the surface of the vehicle is predicted to be fully turbulent, implying that the effects of roughness-induced transition could persist on into the terminal and possibly the landing phases of the mission.

\section{Controls Scheduling in the Presence of Roughness-Induced Transition}

As a means of quantifying the aerodynamic and performance penalties on the vehicle that accrue from the formation of turbulent wedges on its surface, the scheduling of the control surface deflections required to fly the nominal trajectory when accounting for the presence of roughness-induced transition was calculated using the approach described in section VI.B on page 12. The results of this analysis are shown in Fig. 16 on the following page and Fig. 17 on the next page, which focus on that part of the trajectory post transition i.e. from $t=550 \mathrm{~s}$ to $t=820 \mathrm{~s}$. As depicted, the nominal control schedules (in red) are perturbed to some extent in counteracting the additional aerodynamic loads that are induced by the increased viscous stresses acting on the vehicle within the turbulent wedges. Although the resultant perturbations to the nominal deflections seem rather small in most cases, there are some short-lasting events during which the control deflections required to overcome transient moments induced on the vehicle by the turbulence are indeed quite large compared to their nominal values (the tail deflections close to the very end of the trajectory are a case in point), and on the basis of this information a prudent engineer would most likely order a more in-depth characterisation of the unsteady aerodynamic loads on the vehicle before prescribing design limits on the deflections of the control surfaces. To gain an appreciation of the effects of roughness on the performance of the system that result through the direct imposition of the additional viscous loads on the vehicle as well as through the secondary mechanism of the additional aerodynamic force (principally drag) that results from the deflection of the control surfaces, the dispersion of final latitude and longitude that result from calculating the re-entry trajectories with roughness-induced transition accounted for is plotted in Fig. 18. Although the statistics for the performance of the vehicle in the presence of roughness-induced 
transition are extremely sparse as yet, given the computational effort required to generate them even with a reduced-order model, a comparison with the results shown in Fig. 13 on page 17, where only smooth-surface transition was accounted for, is rather illuminating. Although the scatter of the predictions in the two cases is comparable, the data, as sparse as it is, seems to reveal a small but consistent bias towards an increase in the final longitude attained by the vehicle when the presence of roughness-induced transition is accounted for.
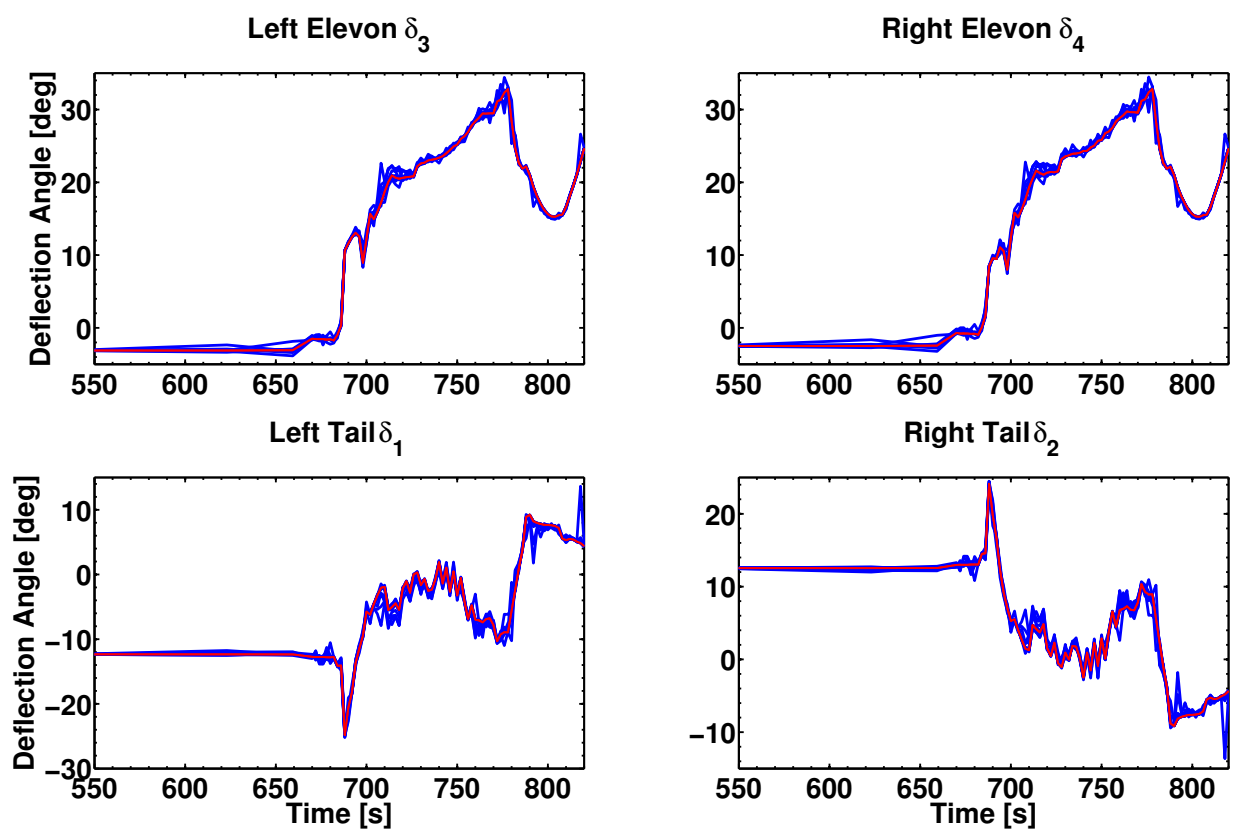

Figure 16: Scheduled deflections of the elevons and rear fins in the presence of roughness-induced boundary layer transition (Blue). Nominal control surface deflections shown in Red.

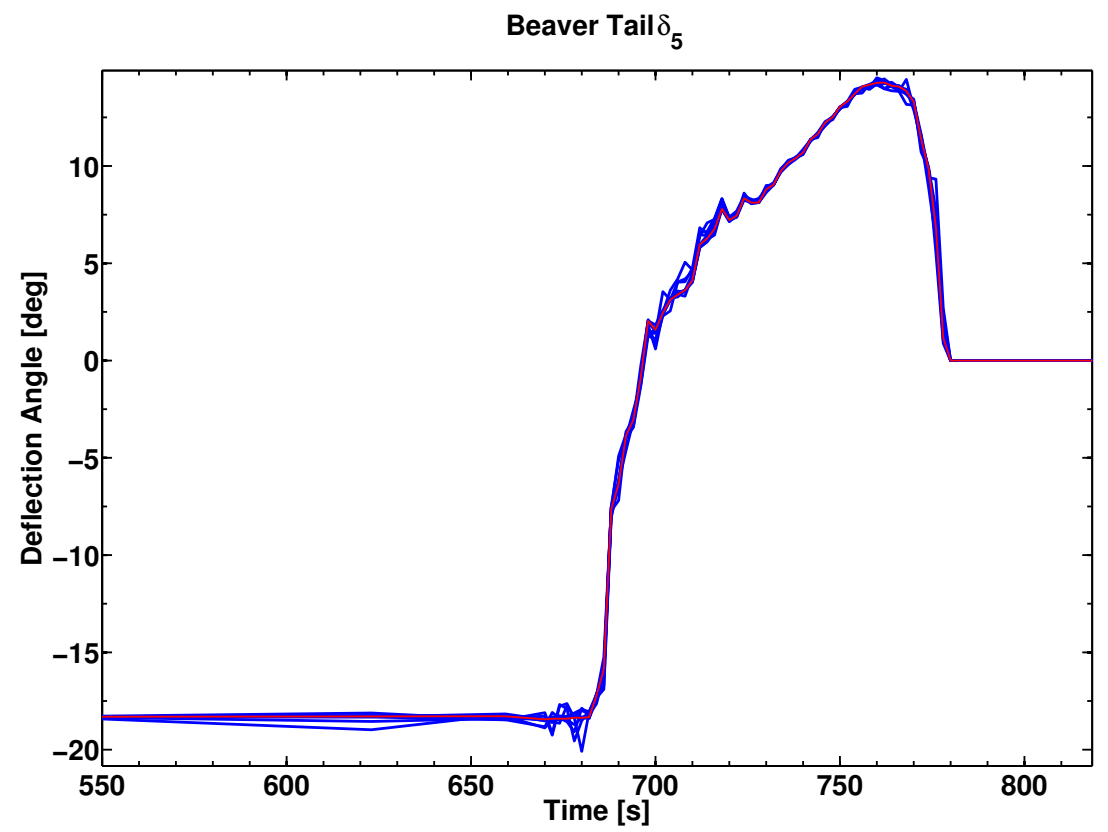

Figure 17: Scheduled deflections of the beaver tail in the presence of roughness-induced boundary layer transition (Blue). Nominal control surface deflections shown in Red. 

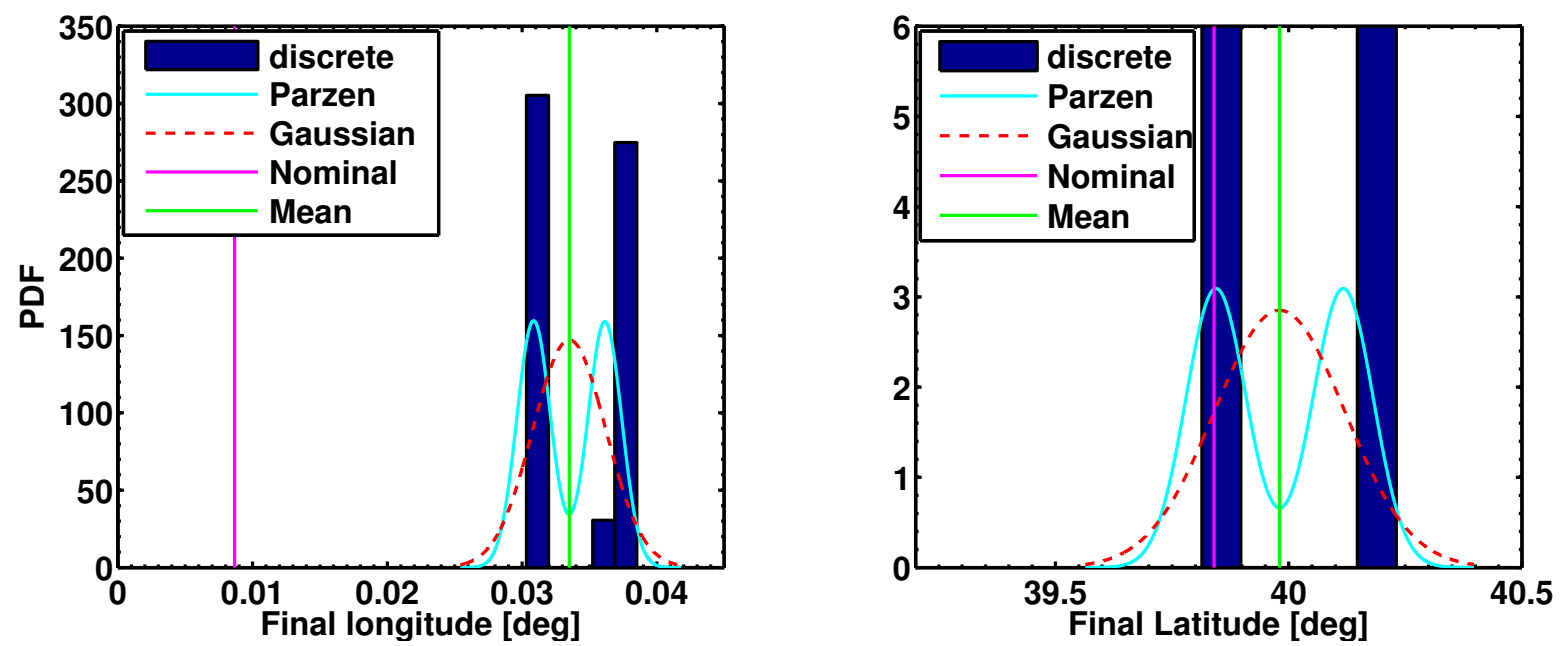

Figure 18: Results of the integration of twenty sample reentry trajectories to estimate the effect of surface roughness-induced transition of the boundary layer on the aerodynamic performance of the CFASTT-1 vehicle, showing the resultant spread of final longitudes and lattitudes about the nominal.

\section{Conclusion}

$\mathrm{T}$ HE present work illustrates the power of reduced-order modelling in examining the sensitivity of the reentry trajectory of a typical reusable launch vehicle to uncertainties in the nature of the transition from laminar to turbulent flow on its surface as it descends through the atmosphere. An engineering method has been developed to account for some of the complex physics associated with roughness-induced boundary layer transition at hypersonic speeds over arbitrary shapes. The method has been developed in order to yield, during the preliminary design phase, an assessment of the sensitivity of any particular vehicle configuration to the aero-thermodynamic effects of boundary layer transition in the presence of uncertainties in the characterisation of the system in terms of its model parameters, and variability in the properties of the atmosphere through which the vehicle might descend. In the present study, it is shown how, for one particular vehicle configuration, not accounting for the deflection of the control surfaces that is required to counteract the aerodynamic moments resulting from asymmetric boundary layer transition may possibly result in under-design of the required control limits for the vehicle, and that the penalty that might be paid for retaining complete controllability of the vehicle throughout its trajectory in the presence of roughnessinduced boundary layer transition may be a compounding of the variability in the integrated aerodynamic forces on the vehicle, which in turn may result in a small but measurable dispersion in the latitude and longitude at which the vehicle reaches the end of its re-entry trajectory. It seems highly likely, however, that re-optimization of the control law for the vehicle while accounting for the uncertainty in the time and position of boundary layer transition will be sufficient to ameliorate this effect.

\section{References}

\footnotetext{
${ }^{1}$ Schneider, S. P., Hypersonic Boundary Layer Transition on Reusable Launch Vehicles. Presented at the RLV/SOV Airframe Technology Review, NASA Langley, 19-22 November 2002.

${ }^{2}$ Berry, S. A., and Horvath, T. J., Discrete Roughness Transition for Hypersonic Flight Vehicles. 45th AIAA Aerospace Sciences Meeting and Exhibit, Reno NV, 8-11 January 2007.

${ }^{3}$ Stetson, K. F., Comments on Hypersonic boundary layer Transition, TR-90-3057, Wright Research and Development Center, Wright Patterson AFB, Dayton OH, September 1990.

${ }^{4}$ Anderson, J. D. Jr., Hypersonic and High Temperature Gas Dynamics, McGraw-Hill Book Company, 1989.

${ }^{5}$ Chen, F., and Malik, M. R., Comparison of Boundary Layer Transition on a Cone and Flat Plate at Mach 3.5, 26th AIAA Aerospace Sciences Meeting, Reno NV, 11-14 January 1988.

${ }^{6}$ Wuilbercq, R., Ahmad, A., Scanlon, T., and Brown, R., Towards Robust Aero-thermodynamic Predictions for Re-usable Singlestage to Orbit Vehicles, 18th AIAA/3AF International Space Planes and Hypersonic Systems and Technologies Conference, Tours, France, 24-28 September 2012.
} 
${ }^{7}$ Meador W. E. and Smart, M., Reference Enthalpy Method developed from Solutions of the Boundary Layer Equations, AIAA Journal, Vol. 43, No. 1, 2005, pp. 135-139.

${ }^{8}$ Hamilton, H. H., II, Greene, F. A., and DeJarnette, F. R., Approximate Method for Calculating Heating Rates on ThreeDimensional Vehicles, Journal of Spacecraft and Rockets, Vol. 31, No. 3, 1994, pp. 345-354.

${ }^{9}$ Cohen-Steiner, D., and Morvan, J. M., Restricted Delaunay Triangulations and Normal Cycle, 19th ACM Symposium on Computational Geometry, San Diego CA, 8-10 June 2003.

${ }^{10}$ Fay, J. A., and Riddell, F. R., Theory of Stagnation Point Heat Transfer in Dissociated Air, Journal of the Aeronautical Sciences, Vol. 25, No. 2, 1958, pp. 73-85.

${ }^{11}$ Bowcutt. K. G. Anderson, D. J., and Capriotti, D., Viscous Optimized Hypersonic Waveriders, 25th AIAA Aerospace Sciences Meeting, Reno NV, 12-15 January 1987.

${ }^{12}$ Bertin, J. J., Hypersonic Aerothermodynamics, AIAA Education Series, 1994.

${ }^{13}$ Hale, N. W. Jr., Lamotte, N. O., and Garner, T. W., Operational Experience with Hypersonic Flight of the Space Shuttle, 11th AIAA/AAAF International Space Planes and Hypersonic Systems and Technology Conference, Orleans, France, 29 September - 4 October 2002.

${ }^{14}$ Reda, D. C., Review and Synthesis of Roughness-Dominated Transition Correlations for Re-entry Applications, Journal of Spacecraft and Rockets, Vol. 39, No. 2, 2002, pp. 161-167.

${ }^{15}$ Ryan K. M. , Lewis M. J., and Yu K. H., Comparison of Robust Optimization Methods Applied to Hypersonic Vehicle Design, AIAA Modeling and Simulation Technologies Conference, Boston MA, 19-22 August, 2013.

${ }^{16}$ Doorly, D. J., and Smith, F. T., Initial-Value Problems for Spot Disturbances in Incompressible or Compressible Boundary Layers, Journal of Engineering Mathematics, Vol. 26, 1992, pp. 87-106.

${ }^{17}$ Vinh, N. X., Busemann, A., and Culp, R. D., Hypersonic and Planetary Entry Flight Mechanics, University of Michigan Press, Ann Arbor, MI, 1980.

${ }^{18}$ Pescetelli, F., Minisci, E., and Brown, R. E., Re-entry Trajectory Optimization for a SSTO vehicle in the Presence of Atmospheric Uncertainties, 5th European Conference for Aeronautics and Space Science (EUCASS), Munich, Germany, 1-4 July, 2013.

${ }^{19}$ Vasile, M., Finite Elements in Time: a Direct Transcription Method for Optimal Control Problems, AIAA/AAS Astrodynamics Specialist Conference, Toronto, Canada, 2-5 August, 2010.

${ }^{20}$ Betts, J. T., Practical Methods for Optimal Control and Estimation Using Nonlinear Programming, Second Edition, SIAM, 2010.

${ }^{21}$ Costa, M., and Minisci, E., MOPED: a Multi-Objective Parzen-based Estimation of Distribution algorithm, 2nd international conference on Evolutionary Multi-criterion Optimization, Turin, Italy, 8-11 April, 2003.

${ }^{22}$ Vasile, M., Minisci, E., and Locatelli, M., An Inflationary Differential Evolution Algorithm for Space Trajectory Optimization, IEEE Transactions on Evolutionary Computation, Vol. 15, No. 2, 2011, pp. 267-281. 